\title{
Simulation of Compressible Two-Phase Flows Using a Void Ratio Transport Equation
}

\author{
Eric Goncalves ${ }^{1, *}$ and Dia Zeidan ${ }^{2}$ \\ ${ }^{1}$ ENSMA, Institut Pprime, UPR 3346 CNRS, 86961 Futuroscope Chasseneuil, France. \\ 2 School of Basic Sciences and Humanities, German Jordanian University, Amman, \\ Jordan.
}

Received 25 January 2017; Accepted (in revised version) 1 November 2017

\begin{abstract}
A compressible and multiphase flows solver has been developed for the study of liquid/gas flows involving shock waves and strong expansion waves leading to cavitation. This solver has a structure similar to those of the one-fluid Euler solvers, differing from them by the presence of a void ratio transport-equation. The model and the system of equations to be simulated are presented. Results are displayed for shock and expansion tube problems, shock-bubble interaction and underwater explosion. Close agreement with reference solutions, obtained from explicit finite volume approaches, is demonstrated. Different numerical methods are additionally displayed to provide comparable and improved computational efficiency to the model and the system of equations. The overall procedure is therefore very well suited for use in general two-phase fluid flow simulations.
\end{abstract}

AMS subject classifications: 35L65, 65M08, 76F55, 76T10, 80A20

Key words: Compressible two-phase flows, cavitation, homogeneous model, shock and expansion waves, inviscid simulation.

\section{Introduction}

Theoretical and numerical modeling of two-phase fluid flow problems is of practical importance in various areas of industry such as thermal power generation plants, propulsion, hydraulic turbines and also environmental applications. Despite their relevance in industrial and environmental applications, compressible two-phase flow investigations have remained complex and challenging areas of applied mathematics and computational methods. The most widely used modeling approach is based on averaged twophase fluid flow model such as the one-fluid formulation. Within such averaged model,

*Corresponding author. Email addresses: eric.goncalves@ensma.fr (E. Goncalves), dia.zeidan@gju.edu.jo (D. Zeidan) 
there are different approaches according to the physical assumptions of interest made on the local mechanical and thermodynamical equilibrium and to the slip condition between phases. This has resulted in the development of diverse models and system of equations ranging from seven to three equations only. The two-fluid approach is the most complete as it represents the dynamics of the quantities mass, momentum, energy and void fraction. One of the most used two-fluid model is the one proposed by Baer and Nunziato [1]. The computation of the two-fluid model is also known to be a real challenge [2-5]. As an alternative way, a reduced five-equation model has been derived with the assumptions of velocity and pressure equilibrium [6-8] and applied with success in various flows. By assuming the thermal equilibrium between phases, a four-equation model can be expressed. It is composed by three conservation laws for mixture quantities completed by an equation for a non-conservative quantity describing the flow topology, usually the void ratio $[9,10]$. The source term includes explicitly the mass transfer between phases. A very popular formulation has been developed to simulate cavitating flows and involves tunable parameters for the vaporization and condensation processes (different sets of parameters are presented in [11]). The domain of variation of these coefficients is huge (from 1 to $10^{6}$ ) leading to serious problems of calibration for different configurations. Moreover, this family of models is not thermodynamically well-posed and does not respect thermodynamic constraints [12]. With the assumption of complete thermodynamic equilibrium between phases (local temperature, pressure and free Gibbs enthalpy equality between phases), the three-equation models or Homogeneous Equilibrium Models (HEM) are derived [13-15].

A critical aspect for two-phase simulations concerns the numerical methods of interest and their accuracy problems. The hyperbolic nature of such flows and their characteristic analysis makes the simulation very stiff and challenging. Large variations of the speed of sound in the mixture is also a difficult problem. Indeed, the speed of sound can be several orders of magnitude higher in the liquid phase than in the two-phase mixture. The non-monotonic behavior of the sound speed in the mixture causes inaccuracies in wave's transmission across interfaces. In addition to that, the volume fraction variation across acoustic waves causes difficulties for the Riemann problem resolution particularly in the derivation of approximate Riemann solvers. This is due to the occurrence of the large discontinuities of thermodynamic variables and equations of state involved at material interfaces. As a result, numerical instabilities and spurious oscillations appear through the complete wave structure [16]. The reason for such unusual behavior lies in the numerical dissipation of the methods which reproduce a thermodynamic path that is not correct. This also implies computational failure for Godunov methods which is due to the large decrease of the pressure up to vacuum ghost.

In the present paper, a four-equation model is considered. The set of equations includes three conservation laws for mixture quantities along with a void ratio transportequation $[10,17]$. The mass transfer between phases appearing explicitly in the formulation is closed by assuming its proportionality with the mixture velocity divergence. This model does not involve any tunable parameters and the thermodynamic coherence is re- 
spected. Due to the source term and the non-conservative form, the numerical integration of the void ratio equation is very stiff. We focus here on the numerical scheme influence on the basis of inviscid applications. The set of equations is solved by means of explicit finite volume techniques based on different schemes such as Jameson-Schmidt-Turkel [18], Rusanov [19], AUSM-type [20], VF Roe [21] and HLLC Riemann solvers methods [22]. This is followed by computational simulations on one-dimensional inviscid problems to study the behavior of the considered numerical methods. Computational results are then displayed for both shock tube and rarefaction problems, including problems of large depression leading to cavitation. Numerical results are validated with reference solutions computed with two-fluid solvers. We also compare the present model with two popular models largely used in cavitation simulations: a barotropic three-equation model and a Kunz-type four-equation model. These test cases establish the ability, accuracy and efficiency of our computational treatment.

The method is also tested on two-dimensional inviscid problems. A shock-bubble interaction and an underwater explosion with cavitation are performed. Comparisons between computational results are proposed.

The paper is organized as follows. In Section 2, we first review the theoretical formulation and models. The description of numerical methods is presented in Section 3. Then, one-dimensional numerical results are proposed and compared with reference solutions in Section 4. In Section 5, two-dimensional cases are considered for the assessment of the proposed formulation. Finally, conclusions and future investigations are discussed.

\section{Governing equations and models}

The homogeneous mixture approach is used to model two-phase flows. The phases are assumed to be sufficiently well mixed and the disperse particle size are sufficiently small thereby eliminating any significant relative motion. The phases are strongly coupled and moving at the same velocity. In addition, the phases are assumed to be in thermal and mechanical equilibrium: they share the same temperature $T$ and the same pressure $P$. The evolution of the two-phase flow can be described by the conservation laws that employ the representative flow properties as unknowns just as in a single-phase problem.

\subsection{A four-equation model}

We consider a reduction of the five-equation Kapila model [6]. We assume that the liquid is at its saturation state. The model consists in three conservation laws for mixture quantities and an additional equation for the void ratio $\alpha$. We present below the inviscid two-dimensional equations, expressed in variables $w=(\rho, \rho \vec{V}, \rho E, \alpha)$ :

$$
\frac{\partial \rho}{\partial t}+\operatorname{div}(\rho \vec{V})=0
$$




$$
\begin{aligned}
\frac{\partial(\rho \vec{V})}{\partial t}+\operatorname{div}(\rho \vec{V} \otimes \vec{V}+P I d) & =0 \\
\frac{\partial(\rho E)}{\partial t}+\operatorname{div}(\rho \vec{V} H) & =0 \\
\frac{\partial \alpha}{\partial t}+\vec{V} \cdot \operatorname{grad}(\alpha) & =\underbrace{\left(\frac{\rho_{l} c_{l}^{2}-\rho_{v} c_{v}^{2}}{\rho_{l} c_{l}^{2}}\right)+\frac{\rho_{v} c_{v}^{2}}{\alpha-\alpha}}_{=K}) \operatorname{div}(\vec{V}),
\end{aligned}
$$

where $\vec{V}=(u, v)$ is the center of mass velocity vector, $E=e+V^{2} / 2$ denotes the mixture total energy and $H=h+V^{2} / 2$ the mixture total enthalpy. The term $K$ involves the speed of sound of pure phases $c_{k}$ and it reflects the effects of changes in volume of each phase.

To close the system, an equation of state (EOS) is necessary to link the pressure and the temperature to the internal energy and the density. For the pure phases, we used the convex stiffened gas EOS (see [23]):

$$
\begin{aligned}
P(\rho, e) & =(\gamma-1) \rho(e-q)-\gamma P_{\infty} \\
P(\rho, T) & =\rho(\gamma-1) C_{v} T-P_{\infty}, \\
T(\rho, h) & =\frac{h-q}{C_{p}}
\end{aligned}
$$

where $\gamma=C_{p} / C_{v}$ is the heat capacity ratio, $C_{p}$ and $C_{v}$ are thermal capacities, $q$ the energy of formation and $P_{\infty}$ is a constant reference pressure. The speed of sound $c$ is given by:

$$
c^{2}=\gamma \frac{P+P_{\infty}}{\rho}=(\gamma-1) C_{p} T .
$$

In the mixture area, an expression for the pressure and the temperature can be deduced from the thermal and mechanical equilibrium assumption [24]. These expressions are available in all possible fluid states, function of the void ratio $\alpha$ and the vapour mass fraction $Y=\alpha \rho_{v} / \rho$ :

$$
\begin{aligned}
P(\rho, e, \alpha, Y) & =(\gamma(\alpha)-1) \rho(e-q(Y))-\gamma(\alpha) P_{\infty}(\alpha), \\
\frac{1}{\gamma(\alpha)-1} & =\frac{\alpha}{\gamma_{v}-1}+\frac{1-\alpha}{\gamma_{l}-1}, \\
q(Y) & =Y q_{v}+(1-Y) q_{l}, \\
P_{\infty}(\alpha) & =\frac{\gamma(\alpha)-1}{\gamma(\alpha)}\left[\alpha \frac{\gamma_{v}}{\gamma_{v}-1} P_{\infty}^{v}+(1-\alpha) \frac{\gamma_{l}}{\gamma_{l}-1} P_{\infty}^{l}\right], \\
T(\rho, h, Y) & =\frac{h_{l}-q_{l}}{C_{p_{l}}}=\frac{h_{v}-q_{v}}{C_{p_{v}}}=\frac{h-q(Y)}{C_{p}(Y)}, \\
C_{p}(Y) & =Y C_{p_{v}}+(1-Y) C_{p_{l}} .
\end{aligned}
$$


The four equations form a system of conservation laws having a hyperbolic nature. The eigenvalues of the system are: $u-c_{\text {wallis }}, u, u, u, u+c_{\text {wallis }}$, where $c_{\text {wallis }}$ is the propagation of acoustic waves without mass and heat transfer [25]. This speed of sound is expressed as a weighted harmonic mean of speeds of sound of each phase:

$$
\frac{1}{\rho c_{\text {wallis }}^{2}}=\frac{\alpha}{\rho_{v} c_{v}^{2}}+\frac{1-\alpha}{\rho_{l} c_{l}^{2}}
$$

\subsection{Mass transfer modelling}

When mass transfer between phases occurs, the expression for the void ratio equation becomes:

$$
\frac{\partial \alpha}{\partial t}+\operatorname{div}(\alpha \vec{V})=(\underbrace{\left(\frac{\rho_{l} c_{l}^{2}-\rho_{v} c_{v}^{2}}{\frac{\rho_{l} c_{l}^{2}}{1-\alpha}+\frac{\rho_{v} c_{v}^{2}}{\alpha}}\right)}_{=K}+\alpha) \operatorname{div}(\vec{V})+\underbrace{\left(\frac{\frac{c_{v}^{2}}{\alpha}+\frac{c_{l}^{2}}{1-\alpha}}{\frac{\rho_{l} c_{l}^{2}}{1-\alpha}+\frac{\rho_{v} c_{v}^{2}}{\alpha}}\right)}_{=1 / \rho_{I} \text { theinterfacialdensity }} \dot{m},
$$

where $\dot{m}$ is the mass transfer between phases.

By assuming that the mass transfer is proportional to the divergence of the velocity, it is possible to build a family of models in which $\dot{m}$ is expressed as (the demonstration is presented in [26])

$$
\dot{m}=\frac{\rho_{l} \rho_{v}}{\rho_{l}-\rho_{v}}\left(1-\frac{c^{2}}{c_{\text {wallis }}^{2}}\right) \operatorname{div}(\vec{V}) .
$$

The speed of sound in the mixture can be expressed as a function of the enthalpy of each phase [10]:

$$
\rho c^{2}=(\gamma(\alpha)-1)\left[\frac{\rho_{v} \rho_{l}}{\left(\rho_{l}-\rho_{v}\right)}\left(h_{v}-h_{l}\right)\right] .
$$

Enthalpies of pure phase $h_{l}$ and $h_{v}$ are computed with the mixture temperature $T$. The system is hyperbolic with the characteristic waves speeds: $u-c, u, u, u, u+c$.

To ensure the thermodynamic coherence, the mixture speed of sound has to vary between the Wallis velocity (without mass transfer) and the equilibrium one evaluated with the assumption of local thermodynamic equilibrium: equalities of pressure, temperature and free Gibbs enthalpy between phases. An expression of the equilibrium speed of sound is given in [12]. Properties as regard to both convexity and mixture speed of sound conditions have been previously studied $[14,26]$.

We assume that the vaporization pressure varies linearly with the temperature:

$$
P_{\text {vap }}(T)=P_{\text {vap }}\left(T_{\text {ref }}\right)+\frac{d P}{d T}\left(T-T_{\text {ref }}\right) .
$$

The constant quantity $d P / d T$ is evaluated with a thermodynamic table. 
The mass transfer term is activated when the local pressure $P$ is smaller than the vapour pressure $P_{\text {vap }}(T)$ evaluated using the relation (2.19). The temperature and pressure relations are coupled and an iterative procedure on the temperature is done with five iterations. For all considered test cases, the temperature difference at the end of the iterative process is smaller than $0.005 \mathrm{~K}$. It has been checked that the numerical solution did not change with a higher number of iteration.

\section{Numerical methods}

In one-dimensional space, the four-equation model can be represented in a matrix form as:

$$
\frac{\partial w}{\partial t}+\frac{\partial(F(w))}{\partial x}=S(w)
$$

Here $w=(\rho, \rho u, \rho E, \alpha)$ is the vector of conserved variables and the void ratio, $F$ the convective flux and $S$ the source term given in (2.16).

Based on finite volume techniques, the computational cells involve the discretization of the spatial domain $x$ into regular meshes of length $\Delta x$ and the temporal domain $t$ into intervals of duration $\Delta t$. A discrete form of equations (3.1) can be written as:

$$
\Delta x \frac{w_{i}^{n+1}-w_{i}^{n}}{\Delta t}+F_{i+1 / 2}^{n}-F_{i-1 / 2}^{n}=S_{i}^{n} \Delta x
$$

where the time step should fulfill the CFL condition in order to guarantee stability requirement and $F_{i+1 / 2}^{n}$ is the numerical flux through the cell interface. This numerical flux can be computed using the solution of the Riemann problem or any other numerical method of interest where the resolution of the Riemann problem is fully numerical.

Various formulations of numerical flux have been proposed to solve multiphase compressible flows. See for instance [27,28], and references therein, for such formulations and extensions. In the present study, we tested and compared different formulations, namely, the Jameson-Schmidt-Turkel scheme, an AUSM-type scheme, the Rusanov scheme, two formulations of HLLC scheme and a VF Roe non-conservative scheme.

\subsection{The Jameson-Schmidt-Turkel scheme}

The space-centered Jameson scheme [18] is stabilized by an artificial viscosity, which includes a second-order dissipation term $D_{2}$ and a fourth-order dissipation term $D_{4}$. Each term involves a tunable numerical coefficient, $k^{(2)}$ and $k^{(4)}$ respectively.

$$
\begin{aligned}
& F_{i+1 / 2}=\frac{1}{2}\left[F\left(w_{i}\right)+F\left(w_{i+1}\right)\right]-|u+c|_{i+1 / 2}\left(\varepsilon_{i+1 / 2}^{(2)} \delta w_{i+1 / 2}+\varepsilon_{i+1 / 2}^{(4)} \delta^{3} w_{i+1 / 2}\right), \\
& \varepsilon_{i+1 / 2}^{(2)}=k^{(2)} \operatorname{Max}\left(v_{p_{i}}, v_{p_{i+1}}, v_{\rho_{i}}, v_{\rho_{i+1}}\right), \quad \varepsilon_{i+1 / 2}^{(4)}=\operatorname{Max}\left[0, k^{(4)}-\varepsilon_{i+1 / 2}^{(2)}\right], \\
& v_{p_{i}}=\frac{\left|P_{i+1}-2 P_{i}+P_{i-1}\right|}{P_{i+1}+2 P_{i}+P_{i-1}}, \quad v_{\rho_{i}}=\frac{\left|\rho_{i+1}-2 \rho_{i}+\rho_{i-1}\right|}{\rho_{i+1}+2 \rho_{i}+\rho_{i-1}} .
\end{aligned}
$$


The coefficient $\varepsilon^{(2)}$ is activated near discontinuities according to the sensor $v$ based on pressure gradients. For two-phase flows, the sensor $v$ is also evaluated with density gradients.

\subsection{An AUSM-type scheme}

The flux formulas of AUSM-type have been tested in the resolution of shock waves and interfaces in multicomponent problems under high density ratio between two phases [20,29-32]. We proposed an AUSM+type formulation following the AUSM+up model proposed by Chang and Liou [20].

We define the interface speed of sound and the interface density as:

$$
c_{1 / 2}=\frac{1}{2}\left(c_{L}+c_{R}\right) \quad \text { and } \quad \rho_{1 / 2}=\frac{1}{2}\left(\rho_{L}+\rho_{R}\right),
$$

where subscripts $L$ and $R$ denote the left and right states with respect to the interface. The left and right Mach numbers are then defined based on this speed of sound as:

$$
M_{L}=\frac{u_{L}}{c_{1 / 2}} \quad \text { and } \quad M_{R}=\frac{u_{R}}{c_{1 / 2}} .
$$

The interface values for Mach number and pressure are defined using splitting functions:

$$
\begin{aligned}
M_{1 / 2} & =M_{(4)}^{+}\left(M_{L}\right)+M_{(4)}^{-}\left(M_{R}\right), \\
P_{1 / 2} & =P_{(5)}^{+}\left(M_{L}\right) P_{L}+P_{(5)}^{-}\left(M_{R}\right) P_{R}+K_{u} P_{(5)}^{+}\left(M_{L}\right) P_{(5)}^{-}\left(M_{R}\right) \rho_{1 / 2} c_{1 / 2}\left(u_{L}-u_{R}\right),
\end{aligned}
$$

where $K_{u}$ is a coefficient set to 0.125 . The expression of splitting functions are given in Appendix A.

A general form of interface mass flux in the AUSM-type scheme is defined as:

$$
(\rho u)_{1 / 2}=c_{1 / 2}\left(\rho_{L} M_{(1)}^{+}\left(M_{1 / 2}\right)+\rho_{R} M_{(1)}^{-}\left(M_{1 / 2}\right)\right)+D p,
$$

where $D p$ is a dissipation term based on pressure difference:

$$
\begin{aligned}
D p & =K_{p} \frac{\Delta M \operatorname{Max}\left(1-\bar{M}^{2}, 0\right)\left(P_{L}-P_{R}\right)}{c_{1 / 2}}, \\
\Delta M & =M_{(4)}^{+}\left(M_{L}\right)-M_{(1)}^{+}\left(M_{L}\right)-M_{(4)}^{-}\left(M_{R}\right)+M_{(1)}^{+}\left(M_{L}\right), \\
\bar{M} & =\frac{1}{2}\left(M_{L}+M_{R}\right),
\end{aligned}
$$

where $K_{p}$ is a coefficient set to 1 .

For the void ratio equation, we introduce the interface velocity:

$$
u_{1 / 2}=c_{1 / 2}\left(M_{(1)}^{+}\left(M_{1 / 2}\right)+M_{(1)}^{-}\left(M_{1 / 2}\right)\right) .
$$


With the four-equation system, the expression of the numerical flux is:

$$
F_{i+1 / 2}=\left(\begin{array}{c}
(\rho u)_{1 / 2} \\
\frac{1}{2}(\rho u)_{1 / 2}\left(u_{L}+u_{R}\right)-\frac{1}{2}\left|(\rho u)_{1 / 2}\right|\left(u_{R}-u_{L}\right)+P_{1 / 2} \\
\frac{1}{2}(\rho u)_{1 / 2}\left(H_{L}+H_{R}\right)-\frac{1}{2}\left|(\rho u)_{1 / 2}\right|\left(H_{R}-H_{L}\right) \\
\frac{1}{2} u_{1 / 2}\left(\alpha_{L}+\alpha_{R}\right)-\frac{1}{2}\left|u_{1 / 2}\right|\left(\alpha_{R}-\alpha_{L}\right)
\end{array}\right)
$$

\subsection{The Rusanov scheme}

The Rusanov's scheme [19] is a central-based Godunov-type method:

$$
F_{i+1 / 2}=\frac{1}{2}\left[F\left(w_{i}\right)+F\left(w_{i+1}\right)-\left|S^{+}\right|\left(w_{i+1}-w_{i}\right)\right],
$$

where the wave speed $S^{+}=\operatorname{Max}\left(u_{L}+c_{L}, u_{R}+c_{R}\right)$ is the maximal eigenvalue evaluated at the cell interface.

The Rusanov scheme is known for its excessive diffusive behavior in presence of a contact discontinuity. To avoid this problem, an anti-diffusive term for the contact discontinuity can be directly added in formulation. A low-diffusive formulation can be proposed by modifying the eigenvalue associated to the contact discontinuity, as proposed in [33]:

$$
F_{i+1 / 2}=\frac{1}{2}\left[F\left(w_{i}\right)+F\left(w_{i+1}\right)\right]-\frac{1}{2}\left(\begin{array}{cccc}
\left|S^{+}\right| & 0 & 0 & 0 \\
0 & \left|S^{+}\right| & 0 & 0 \\
0 & 0 & \left|S_{u}\right| & 0 \\
0 & 0 & 0 & \left|S_{u}\right|
\end{array}\right)\left(w_{i+1}-w_{i}\right),
$$

where $S_{u}=\operatorname{Max}\left(u_{L}, u_{R}\right)$.

With respect to the original scheme, the first two equations, which are related to the acoustic waves, are unchanged, while for the energy equation and the void ratio equation, the diffusive part of the scheme has been reduced. In the following, this scheme will be named LD-Rusanov.

\subsection{A first HLLC scheme}

A HLLC Riemann solver $[22,34,35]$ is investigated for the convective flux density computation. The method considers two averaged intermediate states $w_{L}^{*}$ et $w_{R}^{*}$ separated by the contact wave of speed $S_{M}$. The numerical flux $F_{i+1 / 2}$ at a cell interface can be expressed as:

$$
F_{i+1 / 2}= \begin{cases}F\left(w_{L}\right) & \text { if } S_{L}>0 \\ F\left(w_{L}^{*}\right) & \text { if } S_{L} \leq 0<S_{M} \\ F\left(w_{R}^{*}\right) & \text { if } S_{M} \leq 0 \leq S_{R} \\ F\left(w_{R}\right) & \text { if } S_{R}<0\end{cases}
$$


where $S_{L}$ and $S_{R}$ are referred to the speeds of the smallest and largest waves at the cell interface.

The left $(K=L)$ and right $(K=R)$ states of the variables $w_{K}^{*}$, and corresponding fluxes $F\left(w_{K}^{*}\right)$, are defined by:

$$
\begin{aligned}
& w_{K}^{*}=\left(\begin{array}{c}
\rho_{K}^{*} \\
(\rho u)_{K}^{*} \\
(\rho E)_{K}^{*} \\
\alpha_{K}^{*}
\end{array}\right)=\frac{1}{S_{K}-S_{M}}\left(\begin{array}{c}
\rho_{K}\left(S_{K}-u_{K}\right) \\
(\rho u)_{K}\left(S_{K}-u_{K}\right)+P^{*}-P_{K} \\
(\rho E)_{K}\left(S_{K}-u_{K}\right)+P^{*} S_{M}-P_{K} u_{K} \\
\alpha_{K}\left(S_{K}-u_{K}\right)
\end{array}\right), \\
& F\left(w_{K}^{*}\right)=\left(\begin{array}{c}
\rho_{K}^{*} S_{M} \\
(\rho u)_{K}^{*} S_{M}+P^{*} \\
(\rho E)_{K}^{*} S_{M}+P^{*} S_{M} \\
\alpha_{K}^{*} S_{M}
\end{array}\right),
\end{aligned}
$$

where the pressure $P^{*}$ is given by:

$$
P^{*}=P_{L}+\rho_{L}\left(u_{L}-S_{L}\right)\left(u_{L}-S_{M}\right)=P_{R}+\rho_{R}\left(u_{R}-S_{R}\right)\left(u_{R}-S_{M}\right) .
$$

And the contact-wave speed $S_{M}$ is defined by:

$$
S_{M}=\frac{P_{R}-P_{L}+\rho_{L} u_{L}\left(S_{L}-u_{L}\right)-\rho_{R} u_{R}\left(S_{R}-u_{R}\right)}{\rho_{L}\left(S_{L}-u_{L}\right)-\rho_{R}\left(S_{R}-u_{R}\right)} .
$$

The HLLC solver requires the estimates of wave speeds $S_{L}$ and $S_{R}$ in the Riemann problem. A direct and simple wave speed estimation is used:

$$
S_{L}=\operatorname{Min}\left(u_{L}-c_{L}, u_{R}-c_{R}\right) \quad \text { and } \quad S_{R}=\operatorname{Max}\left(u_{L}+c_{L}, u_{R}+c_{R}\right) .
$$

In the following, this scheme will be named HLLC v1.

\subsection{A second HLLC formulation}

Another discretization is proposed based on the following general form of the system:

$$
\frac{\partial U}{\partial t}+\frac{\partial G(U)}{\partial x}+B(U) \frac{\partial u}{\partial x}=0
$$

with

$$
U=\left(\begin{array}{c}
w \\
\alpha
\end{array}\right), \quad G(U)=\left(\begin{array}{c}
F(w) \\
\alpha u
\end{array}\right), \quad \text { and } \quad B(U)=\left(\begin{array}{c}
0 \\
-K-\alpha
\end{array}\right) .
$$

The conservative part is discretized used the flux $F\left(w_{K}^{*}\right)$ similarly to the first formulation v1. The non-conservative part is discretized following the idea of Daude et al. [8]. The term $B(U)$ is approximated with the following relation:

$$
\int_{x_{i-1 / 2}}^{x_{i+1 / 2}} B(U) \frac{\partial u}{\partial x} d x \simeq B\left(U_{i}\right)\left(u_{i+1 / 2}-u_{i-1 / 2}\right) .
$$


The cell interface value $u_{i+1 / 2}$ is expressed as:

$$
u_{i+1 / 2}= \begin{cases}u_{L} & \text { if } S_{L}>0 \\ \frac{S_{L}-u_{L}}{S_{L}-S_{M}} S_{M} & \text { if } S_{L} \leq 0<S_{M} \\ \frac{S_{R}-u_{R}}{S_{R}-S_{M}} S_{M} & \text { if } S_{M} \leq 0 \leq S_{R} \\ u_{R} & \text { if } S_{R}<0\end{cases}
$$

In the following, this scheme will be named HLLC v2.

Second- and third-order accuracy in space can be obtained using the MUSCL approach proposed by van Leer for the construction of $U_{L}$ and $U_{R}$. The minmod slope limiter is used with the MUSCL extrapolation.

\subsection{A VFRoe ncv scheme}

The VFRoe non-conservative scheme is an approximate Riemann solver introduced in $[21,36,37]$. Finding a matrix satisfying Roe's condition may be difficult for two-phase problems with complex EOS. This fact has motivated the development of an alternative to the Roe scheme. The scheme is based on the resolution of linearized Riemann problems written in non-conservative variables. It admits entropy-violating stationary discontinuities, we switch on the Rusanov scheme in this case. form:

Considering the change of variables $w \rightarrow W(w)$, the system reads in non-conservative

$$
\frac{\partial W}{\partial t}+B(W) \frac{\partial Y}{\partial x}=0
$$

where $B$ is the matrix of the transformed system.

At each interface, we solve the following linearized Riemann problem:

$$
\frac{\partial W}{\partial t}+B(\tilde{W}) \frac{\partial W}{\partial x}=0 \text { with } \begin{cases}W(x, 0)=W_{L} & \text { if } x<0 \\ W(x, 0)=W_{R} & \text { if } x>0\end{cases}
$$

where $\tilde{W}$ is an average state depending on $W_{L}$ and $W_{R}$; here we use the simple arithmetic average. The matrix $B(\tilde{W})$ is diagonalizable with real eigenvalues $\tilde{\lambda}_{i}$. We note $\tilde{r}_{i}$ and $\tilde{l}_{i}$ the right and left eigenvectors respectively. The difference $W_{R}-W_{L}$ is projected directly into the space spanned by a linear combination of the right eigenvectors:

$$
W_{R}-W_{L}=\sum_{i=1}^{p} \tilde{\alpha}_{i} \tilde{r}_{i}, \quad \text { with } \quad \tilde{\alpha}_{i}={ }^{t} \tilde{l}_{i} \cdot\left(W_{R}-W_{L}\right) .
$$

The solution of the Riemann problem is composed of constant states separated by a fan of $p$ characteristic lines:

$$
W\left(\frac{x}{t} ; W_{L}, W_{R}\right)= \begin{cases}W_{L} & \text { if } x<\tilde{\lambda}_{1} t \\ W_{k}=W_{L}+\sum_{i=1}^{k} \tilde{\alpha}_{i} \tilde{r}_{i} & \text { if } \tilde{\lambda}_{k} t<x<\tilde{\lambda}_{k+1} t \\ W_{R} & \text { if } x>\tilde{\lambda}_{p} t .\end{cases}
$$


If we suppose that no eigenvalue vanishes, we note $W^{*}$ the approximate state at the interface, i.e. for $\frac{x}{t}=0$. The numerical flux is given by:

$$
F_{i+1 / 2}=F\left(w\left(W^{*}\right)\right) .
$$

For the considered four-equation model, we choose for the non-conservative variable ${ }^{t} W=(\tau, u, P, Y)$ where $\tau=\frac{1}{\rho}$ and $Y=\frac{\alpha \rho_{v}}{\rho}$ is the mass fraction. Note that we have not tested other choices for $W$. The expression of matrix $B$ and associated eigenvectors are given in Appendix B.

\subsection{Treatment of the source term}

The numerical simulations of the initial-boundary value problems are accomplished using splitting approach (except for the HLLC v2 formulation). One starts in solving the source-free homogeneous part of the whole system:

$$
\frac{\partial w}{\partial t}+\operatorname{div}(F(w))=0 .
$$

This is followed by solving the system of ordinary differential equations describing the mass transfer between phases to obtain the complete solution:

$$
\frac{d w}{d t}=S(w)
$$

A first order method is used for this step.

\subsection{Inlet and outlet boundary conditions}

The numerical treatment of the boundary conditions is based on the use of the characteristic relations of Euler equations. The number of variables to impose at boundaries is given by the number of positive characteristics directed into the domain of interest. The characteristic relations obtained for the 4-equation system, in two-dimensional flows, are:

$$
\begin{aligned}
-c^{2}\left(\rho^{c}-\rho^{s}\right)+\left(P^{c}-P^{s}\right) & =0, \\
V_{t}^{c}-V_{t}^{s} & =0, \\
\rho\left(\alpha^{c}-\alpha^{s}\right)-K\left(\rho^{c}-\rho^{s}\right) & =0, \\
\left(P^{c}-P^{s}\right)+\rho c\left(V_{n}^{c}-V_{n}^{s}\right) & =0, \\
\left(P^{c}-P^{s}\right)-\rho c\left(V_{n}^{c}-V_{n}^{s}\right) & =0 .
\end{aligned}
$$

The variables with superscript $c$ denote the variables to be computed at the boundary. Variables with superscript $s$ denote the variables obtained by the current numerical scheme. $V_{t}$ and $V_{n}$ are the tangential and the normal component of the mean velocity, respectively. 
At inflow, we impose the initial values of the void ratio, densities of pure phases and the velocity. The pressure is evaluated with the relation (3.27) and all variables can be evaluated at the boundary.

At outflow, the static pressure is imposed. The variables are computed with four characteristic relations (3.23)-(3.26).

\section{Computational results on one-dimensional two-phase problems}

In this section we exhibit the ability of the current four-equation model, convergence and computational performance of the proposed numerical methods on two groups of two-phase flow problems. In the first group, we considered two shock tube problems to validate the current numerical tool. A comparison with solutions provided with a sevenequation model using the Discrete Equations Method (DEM) is proposed [38]. In DEM approach, the pure fluids are first integrated at the microscopic level and then the discrete formulae are averaged. The obtained continuous model of multiphase flow is equivalent to the Baer-Nunziato model. The infinite rate relaxation procedures are used to correctly treat the full system. The second group tests the expansion tube, double rarefaction, problems which are very stiff cases for numerical methods. Results of the expansion tube problems are validated with other models as we shall see later.

For all cases, values of artificial dissipation coefficients $\left(k^{(2)}, k^{(4)}\right)$ for the Jameson scheme and numerical coefficients $\left(K_{u}, K_{p}\right)$ for the AUSM scheme are given in Table 1.

Table 1: Numerical coefficients for the Jameson and AUSM-type schemes.

\begin{tabular}{||llllll||}
\hline & shock tube & shock tube & \multicolumn{3}{c||}{ double rarefaction tubes } \\
& water-gas & epoxy-spinel & no cavitation & cavitation & cavitation \\
\hline Jameson $\left(k^{(2)}, k^{(4)}\right)$ & $1-0.02$ & $1-0.06$ & $1-0.016$ & $1-0.016$ & $3-0.016$ \\
$\operatorname{AUSM}\left(K_{p}, K_{u}\right)$ & $1-0.125$ & $1-0.125$ & $2-0.2$ & - & - \\
\hline
\end{tabular}

\subsection{Water-gas mixture shock tube}

This test case is proposed in [7], computed with five- and seven-equation models. A one meter shock tube involves a discontinuity of the volume fraction. For $x<0.7$ the gas volume fraction is 0.2 , while it is 0.8 otherwise. The fluids are governed by the stiffened gas EOS and are initially at rest. The left chamber contains high pressure fluids $\left(10^{9} \mathrm{~Pa}\right)$ while the right one contains low pressure fluids $\left(10^{5} \mathrm{~Pa}\right)$. The parameters of EOS are:

$$
\left(\begin{array}{c}
\gamma \\
P_{\infty} \\
\rho
\end{array}\right)_{\text {Liq }}=\left(\begin{array}{c}
4.4 \\
6 \times 10^{8} \mathrm{~Pa} \\
1000 \mathrm{~kg} / \mathrm{m}^{3}
\end{array}\right) \quad \text { and } \quad\left(\begin{array}{c}
\gamma \\
P_{\infty} \\
\rho
\end{array}\right)_{\text {Gas }}=\left(\begin{array}{c}
1.4 \\
0 \mathrm{~Pa} \\
1 \mathrm{~kg} / \mathrm{m}^{3}
\end{array}\right) .
$$



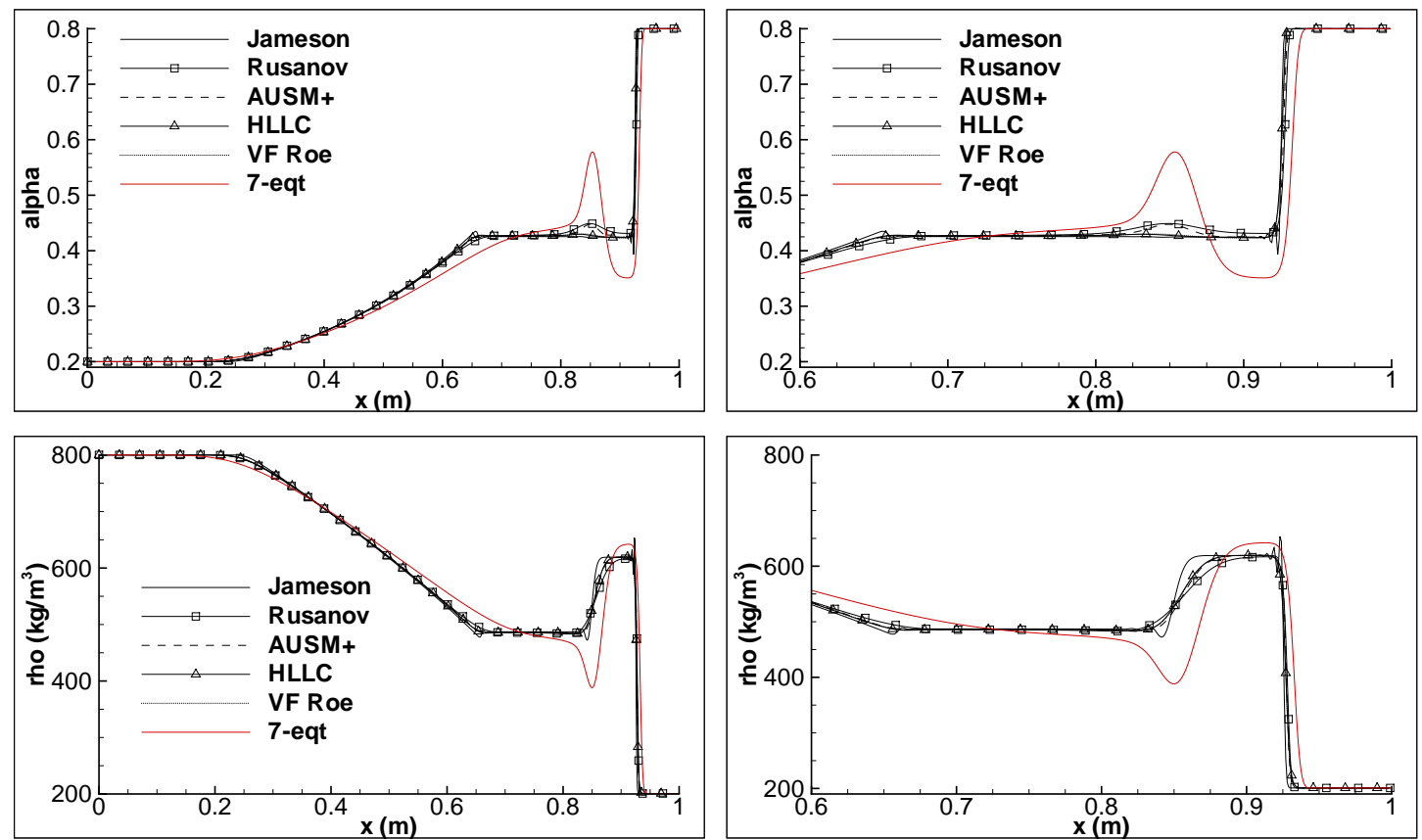

Figure 1: Water-gas shock tube problem, numerical schemes comparison, mesh 1000 cells, $t=0.2$ ms. Void ratio and mixture density profiles along the tube (left), enlargement of these quantities between abscissa 0.6-1 m (right).

Computations have been performed with a mesh of 1000 cells and with a time step of $10^{-7} \mathrm{~s}$. Results are shown at time $0.2 \mathrm{~ms}$. Both versions of the HLLC scheme provided similar results, only the HLLC v1 solution is presented. For this case, the analytical solution has not been evaluated according to the method proposed in [39].

Profiles of void ratio and mixture density are plotted in Fig. 1 for all numerical schemes. An enlargement of solutions are proposed in the post-shock area (on the right). Near discontinuities, the Jameson scheme produced small oscillations of the solution. For the void ratio profile, we observe a small discrepancy in the post-shock area around $x=0.85 \mathrm{~m}$. The solution obtained with the Rusanov and AUSM schemes present a small variation, not captured by other methods. In comparison with the seven-equation model, we notice some differences between the solutions in the volume fraction profile. In particular, the post-shock values of the void ratio are not the same and the seven-equation model shows an oscillation near the contact discontinuity zone. This behaviour was also noted in simulations presented in [7].

Profiles of mixture pressure and velocity are presented in Fig. 2 for all numerical schemes. Results are quite similar. An inset of the post-shock area is shown (on the right). As observed previously, the Jameson solution involves oscillations near the shock area. 

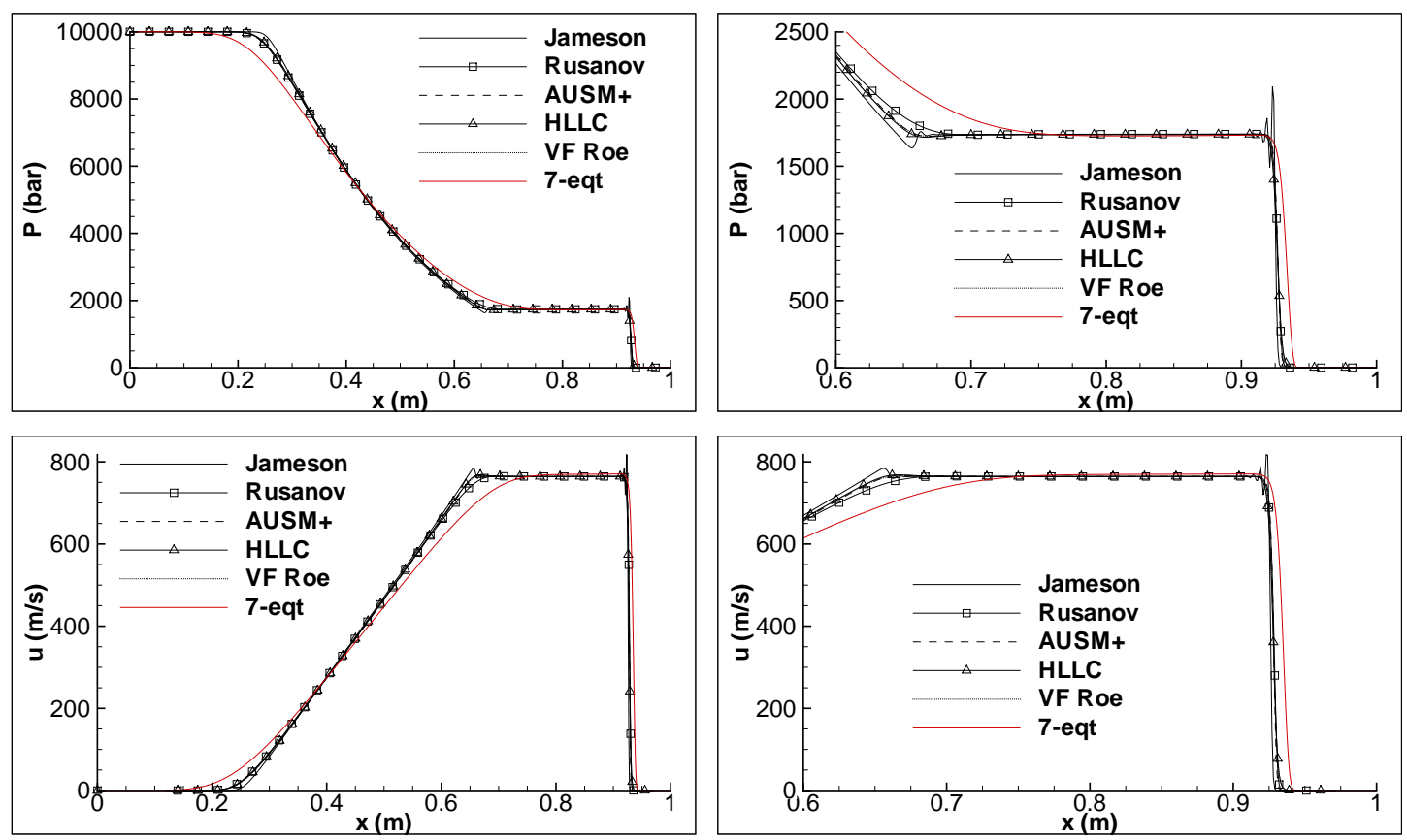

Figure 2: Water-gas shock tube problem, numerical schemes comparison, mesh 1000 cells, $t=0.2$ ms. Mixture pressure and velocity profiles along the tube (left), enlargement of these quantities between abscissa $0.6-1 \mathrm{~m}$ (right).

\subsection{Epoxy-spinel mixture shock tube}

In [39] a one meter tube contains two chambers separated at $x=0.6 \mathrm{~m}$. A mixture of epoxy and spinel fills both chambers. The initial volume fraction of epoxy is 0.5954 everywhere. The left chamber pressure is $2.10^{11} \mathrm{~Pa}$, while the right chamber is at atmospheric pressure. The fluids are governed by the stiffened gas EOS are initially at rest. EOS parameters are:

$$
\left(\begin{array}{c}
\gamma \\
P_{\infty} \\
\rho
\end{array}\right)_{\text {Epoxy }}=\left(\begin{array}{c}
2.43 \\
5.3 \times 10^{9} \mathrm{~Pa} \\
1185 \mathrm{~kg} / \mathrm{m}^{3}
\end{array}\right) \quad \text { and } \quad\left(\begin{array}{c}
\gamma \\
P_{\infty} \\
\rho
\end{array}\right)_{\text {spinel }}=\left(\begin{array}{c}
1.62 \\
141 \times 10^{9} \mathrm{~Pa} \\
3622 \mathrm{~kg} / \mathrm{m}^{3}
\end{array}\right) \text {. }
$$

Computations have been performed on a mesh of 1000 cells and with a time step of $10^{-7} \mathrm{~s}$. Results are shown at time $t=29 \mu \mathrm{s}$. As previously, both versions of the HLLC scheme provided similar results, only the HLLC v1 solution is presented. The analytical solution of the equilibrium model proposed in [39] is added.

Profiles of void ratio and mixture density are illustrated in Fig. 3 for all numerical schemes. An enlargement of solutions are proposed in the post-shock area (on the right). In comparison with the analytical solution, discrepancies appear on the void ratio and density jumps at shock front, which are over-estimated by all methods, especially the 

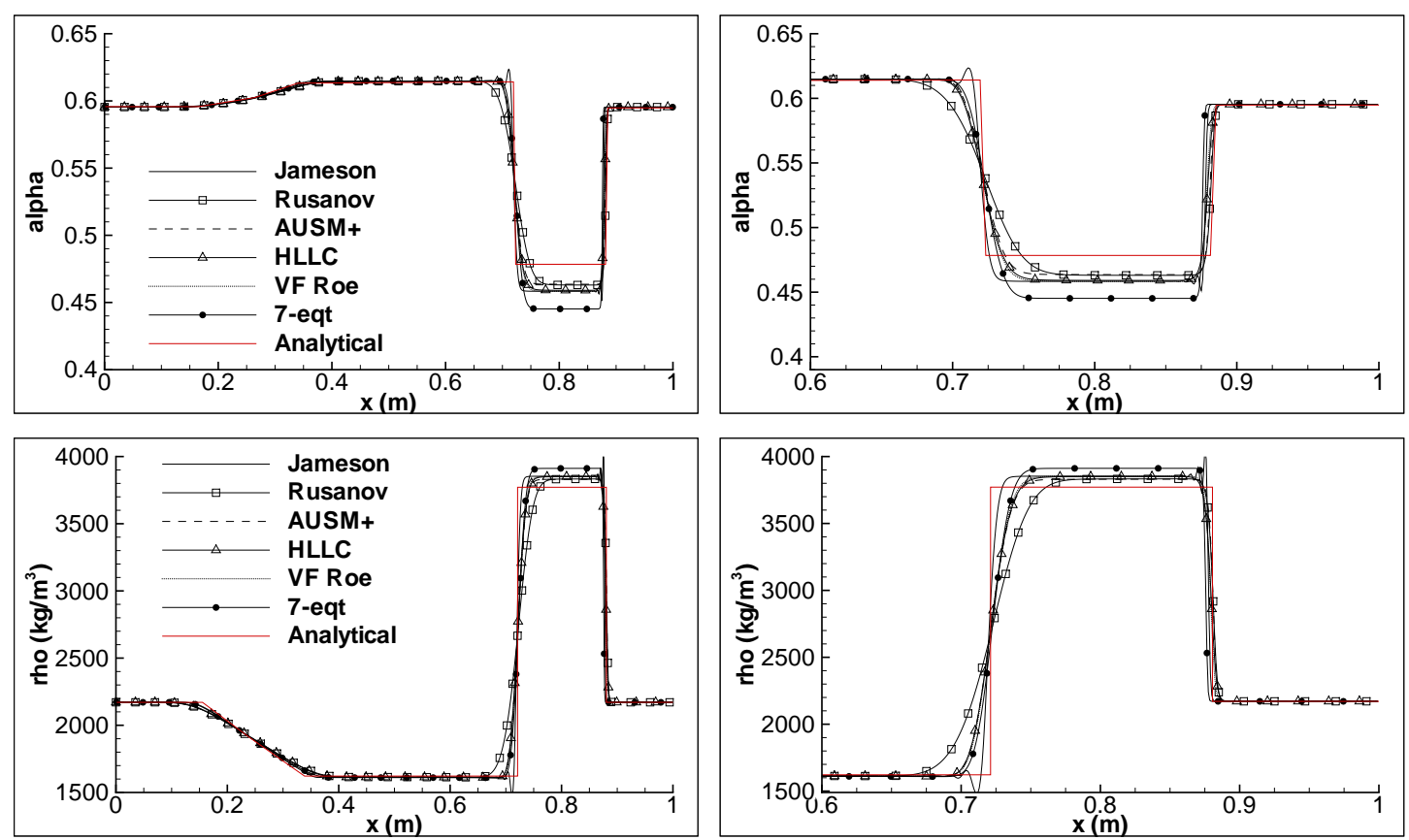

Figure 3: Epoxy-spinel shock tube problem, numerical schemes comparison, mesh 1000 cells, $t=0.29$ ms. Void ratio and mixture density profiles along the tube (left), enlargement of these quantities between abscissa 0.6-1 m (right).

seven-equation model. For the void ratio profiles, the plateau after the shock is less intense using the Rusanov scheme.

Profiles of mixture pressure and velocity are presented in Fig. 4 for all numerical schemes. An inset of the post-shock area is shown (on the right). Differences between solutions are weak. For all schemes, the pressure profiles are in close agreement with the analytical solution. As previously, the solution computed with the Jameson scheme presents small oscillations near discontinuities.

\subsection{Water-gas mixture expansion tube, $\left|u_{0}\right|=2 \mathrm{~m} / \mathrm{s}$}

A double rarefaction tube problem is considered with an initial velocity discontinuity located at the middle of the tube. This test consists in a one meter long tube filled with liquid water at atmospheric pressure and with density $\rho_{l}=1150 \mathrm{~kg} / \mathrm{m}^{3}$. A weak volume fraction of vapor $\alpha=0.01$ is initially added to the liquid. The initial discontinuity is set at $0.5 \mathrm{~m}$, the left velocity is $-2 \mathrm{~m} / \mathrm{s}$ and the right velocity is $2 \mathrm{~m} / \mathrm{s}$, as illustrated in Fig. 5 .

The solution involves two expansion waves. As gas is present, the pressure cannot become negative. To maintain positive pressure, the gas volume fraction increases due to the gas mechanical expansion and creates a pocket [24]. The fluids are governed by the 

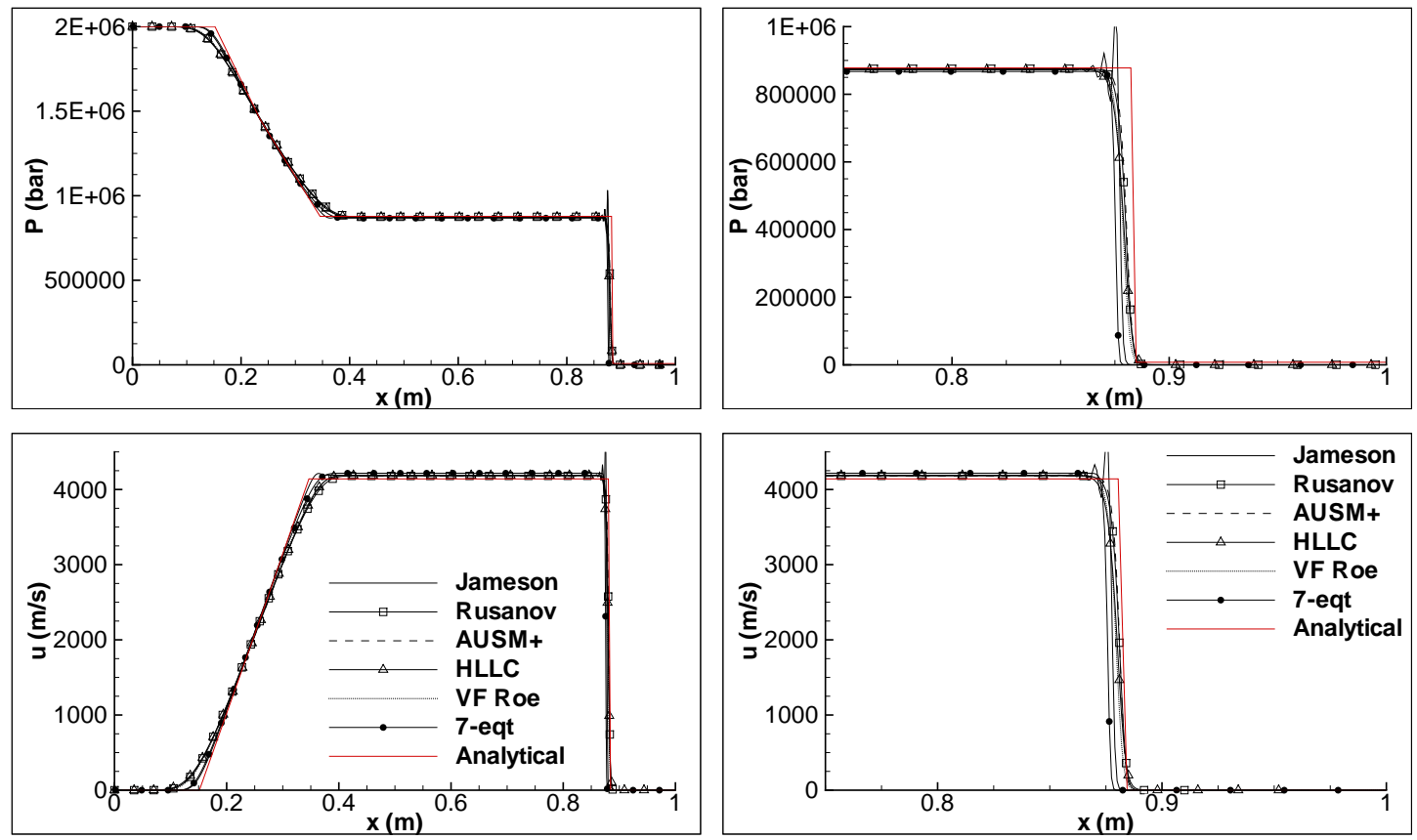

Figure 4: Epoxy-spinel shock tube problem, numerical schemes comparison, mesh 1000 cells, $t=0.29 \mathrm{~ms}$. Mixture pressure and velocity profiles along the tube (left), enlargement of these quantities between abscissa $0.75-1 \mathrm{~m}$ (right).

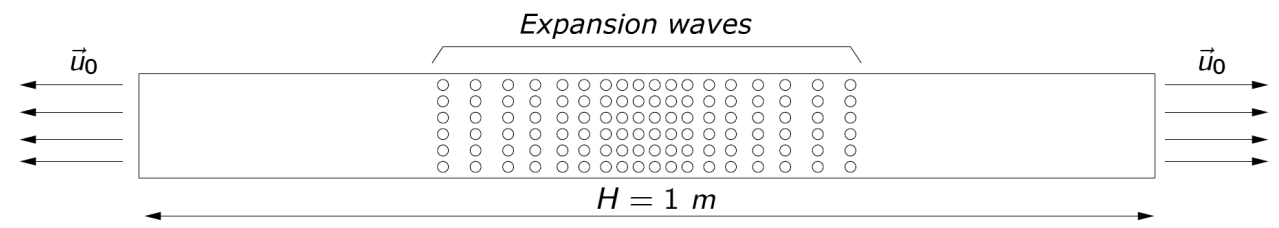

Figure 5: Schematic view of the double rarefaction test case.

stiffened gas EOS, for which parameters are:

$$
\left(\begin{array}{c}
\gamma \\
P_{\infty} \\
\mathrm{q} \\
\rho \\
C_{p}
\end{array}\right)_{\text {Liq }}=\left(\begin{array}{c}
2.35 \\
10^{9} \mathrm{~Pa} \\
-0.1167 \times 10^{7} \mathrm{~J} / \mathrm{kg} \\
1150 \mathrm{~kg} / \mathrm{m}^{3} \\
4267 \mathrm{~J} / \mathrm{K} . \mathrm{kg}
\end{array}\right) ; \quad\left(\begin{array}{c}
\gamma \\
P_{\infty} \\
\mathrm{q} \\
\rho \\
C_{p}
\end{array}\right)_{\text {Gas }}=\left(\begin{array}{c}
1.43 \\
0 \mathrm{~Pa} \\
0.2030 \times 10^{7} \mathrm{~J} / \mathrm{kg} \\
1 \mathrm{~kg} / \mathrm{m}^{3} \\
1487 \mathrm{~J} / \mathrm{K} . \mathrm{kg}
\end{array}\right) .
$$

The mesh contains 1000 cells and the time step is set to $10^{-7} \mathrm{~s}$. Solutions are compared at time $t=3.2 \mathrm{~ms}$ with the two-fluid solution proposed by Zein et al. [40]. Profiles of void ratio, density, pressure and velocity are plotted in Fig. 6 for all numerical schemes except HLLC schemes. The pressure evolution marks large discrepancies. Solutions provided by the Jameson, Rusanov and AUSM schemes are in close agreement with the 

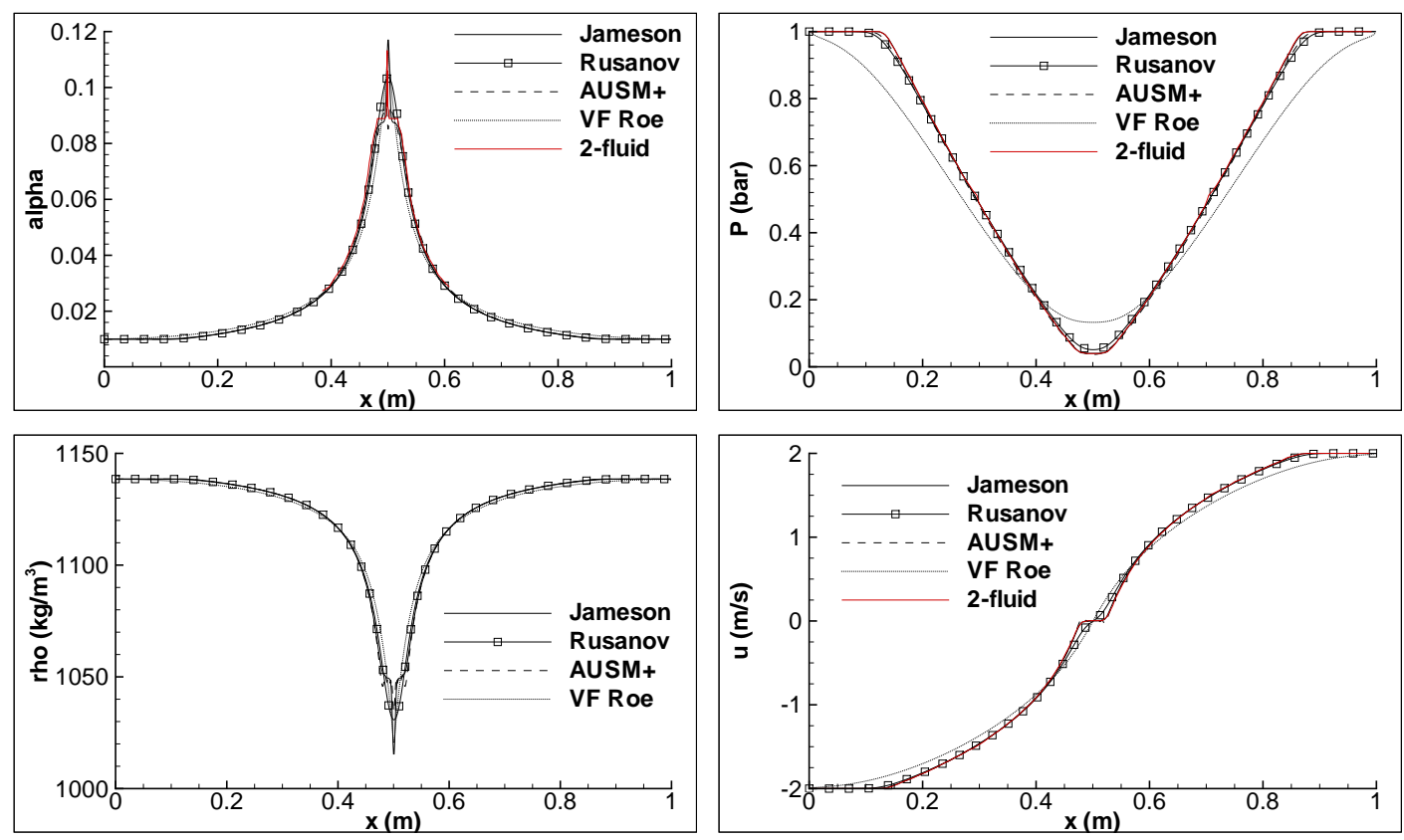

Figure 6: Water-gas double rarefaction $\left|u_{0}\right|=2 \mathrm{~m} / \mathrm{s}$, numerical schemes comparison, mesh 1000 cells, $t=3.2$ ms. Void ratio, mixture density, pressure and velocity profiles.
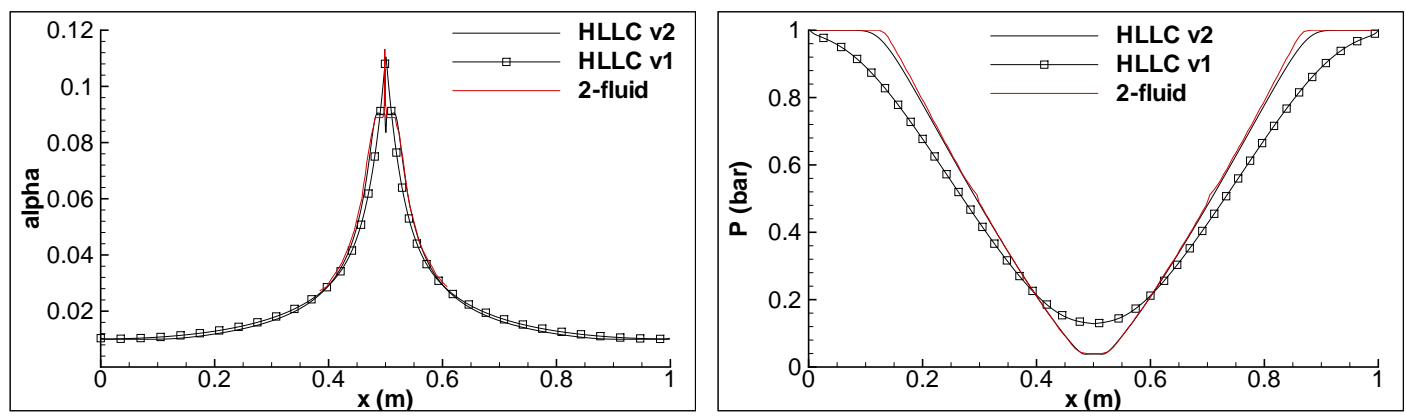

Figure 7: Water-gas double rarefaction $\left|u_{0}\right|=2 \mathrm{~m} / \mathrm{s}$, HLLC schemes comparison, mesh 1000 cells, $t=3.2 \mathrm{~ms}$. Void ratio and mixture pressure profiles.

two-fluid solution. Using the VF Roe ncv scheme, the rarefaction waves are badly predicted. Maybe a different choice for the primitive variable $W$ or another discretization of the non-conservative equation would improve the behaviour of this scheme.

As regard to the computational cost, a CPU time of $14 \mathrm{~h}$ was necessary for the twofluid simulation due to the relaxation procedures. With our four-equation model, the CPU time is only around five minutes.

The two HLLC formulations are now compared. The void ratio and mixture pressure profiles are shown in Fig. 7. Similarly to the VF Roe solution, the rarefaction waves are badly simulated with the first HLLC formulation. On the contrary, the second formula- 
tion provides a solution in close agreement with the two-fluid solution. The discretization of the non-conservative void ratio transport equation is a key point for this Riemann solver.

\subsection{Water-gas mixture expansion tube with cavitation, $\left|u_{0}\right|=2 \mathrm{~m} / \mathrm{s}$}

We consider the same case presented previously adding the mass transfer term. Liquid water is expanded until the saturation pressure is reached then evaporation appears and a cavitation pocket is created. The solution with phase transition is composed of four expansion waves. The extra two expansion waves correspond to the evaporation fronts.

The vapour pressure at the considered reference temperature is $P_{\text {vap }}=51000 \mathrm{~Pa}$. The constant $d P / d T$ evaluated using a thermodynamic table is set to $2044 \mathrm{~Pa} / \mathrm{K}$. The mesh contains 1000 cells and the time step is set to $10^{-7} \mathrm{~s}$. Solutions are compared at time $t=3.2$ ms with the two-fluid solution proposed by Zein et al. [40].

First, we propose a comparison of the considered numerical schemes. For this case, it was not possible to obtain a solution using the VF Roe, HLLC v1 and AUSM+up schemes (even by modifying numerical coefficients $K_{u}$ and $K_{p}$ for the AUSM scheme). The thirdorder formulation is tested for the HLLC v2 scheme, named HLLC 3o in the following.

Profiles of void fraction (in logarithmic scale), pressure, mass fraction and velocity are plotted in Fig. 8 for all schemes. Concerning the mass fraction $Y$, an enlargement of the mid-tube area is proposed, illustrating the vaporization phenomenon. In comparison with the previous case where the void ratio reached values around $10 \%$, values are now close to $70 \%$ in the cavitation pocket. For both pressure and velocity profiles, all numerical results are quite similar in close agreement with the 2-fluid solution. The plateau of pressure at the vapor pressure value is well illustrated.

Discrepancies are noticeable for the maximum values of both the volume and mass fractions of gas. The most extreme results are obtained using the Rusanov and Jameson schemes for which a factor 2 is observed, lower and higher, respectively. The third-order HLLC scheme provides a solution is close agreement with the two-fluid result whereas the first-order formulation under-estimates the maximum value.

Secondly, we compare the present model with two popular models largely used in cavitation simulations: a barotropic model $[14,41]$ and a Kunz-type void ratio transport model [9]. We evaluate the ability of these models to reproduce correctly the cavitation pocket and all waves involved in the physics. The formulation of the barotropic model is given in Appendix C. With this model, it is not possible to start the simulation from a liquid/gas mixture. The model can create a cavitation pocket only from a liquid. The Kunz-type formulation is detailed in Appendix D. This model involves two tunable parameters $C_{\text {prod }}$ and $C_{d e s}$. In the test case, only an evaporation process occurs therefore the constant $C_{\text {des }}$ is set to 0 . The calibration of $C_{\text {prod }}$ was performed in order to fit the reference solution of Zein et al. [40] for the void ratio profile. The closest result was obtained with $C_{\text {prod }}=100 \mathrm{~m}^{3} / \mathrm{kg}$ (we do not present the calibration study). All simulations with the three models were performed using the Rusanov scheme. 

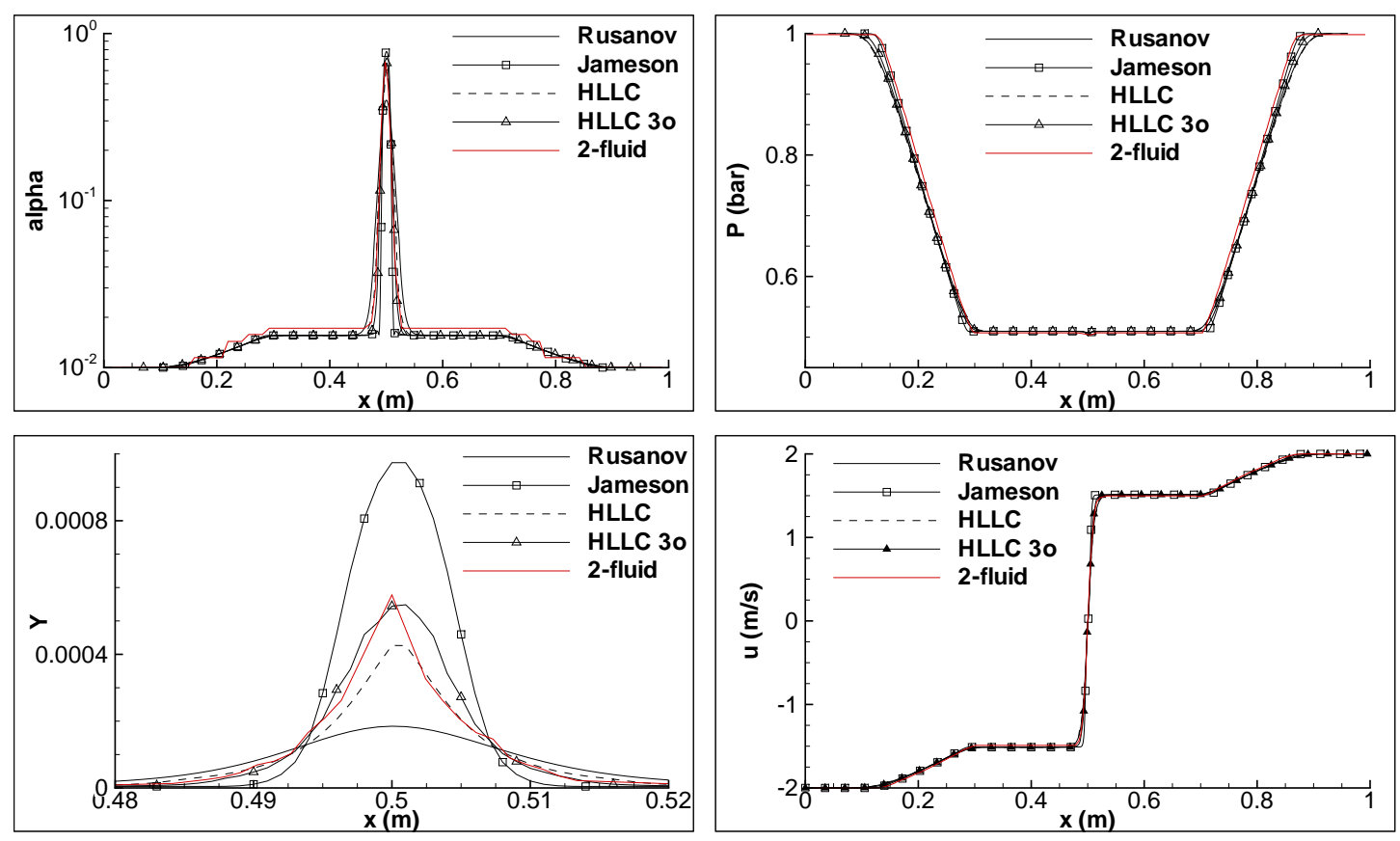

Figure 8: Water-gas double rarefaction with cavitation $\left|u_{0}\right|=2 \mathrm{~m} / \mathrm{s}$, numerical schemes comparison, mesh 1000 cells, $t=3.2 \mathrm{~ms}$. Void ratio, pressure and velocity profiles along the tube, enlargement of the mass fraction between abscissa $0.48-0.52 \mathrm{~m}$.

Profiles of void ratio (in logarithmic scale), pressure, mass fraction of vapor and velocity are plotted in Fig. 9 for the three considered models. The Kunz-type model predicts a cavitation pocket with correct values of void ratio and mass fraction. Yet, the variation of void ratio across the rarefaction waves is not simulated. Both the pressure and velocity evolutions show that rarefaction waves are badly computed. Similarly, the 3-equation model is unable to correctly predicts the rarefaction waves. Moreover, the evaporation fronts and the cavitation pocket are badly reproduced. This is due to the initial condition of the simulation, started from a pure liquid.

\subsection{Water-gas mixture expansion tube with cavitation, $\left|u_{0}\right|=100 \mathrm{~m} / \mathrm{s}$}

The same conditions are used except regarding velocities which are set to $\left|u_{0}\right|=100$ $\mathrm{m} / \mathrm{s}$. The EOS parameters are similar. Due to the large value of the velocity discontinuity, evaporation is much more intense resulting in a large cavitation pocket where the gas volume fraction is close to 1 . However, this pocket does not contain pure gas but a mixture at thermodynamic equilibrium. The quantity $d P / d T$ evaluated with a thermodynamic table is set to $300 \mathrm{~Pa} / \mathrm{K}$. The mesh contains 1000 cells and the time step is set to $10^{-7} \mathrm{~s}$. Solutions are compared at time $t=1.5 \mathrm{~ms}$ with the two-fluid solution proposed by Zein et al. [40]. 

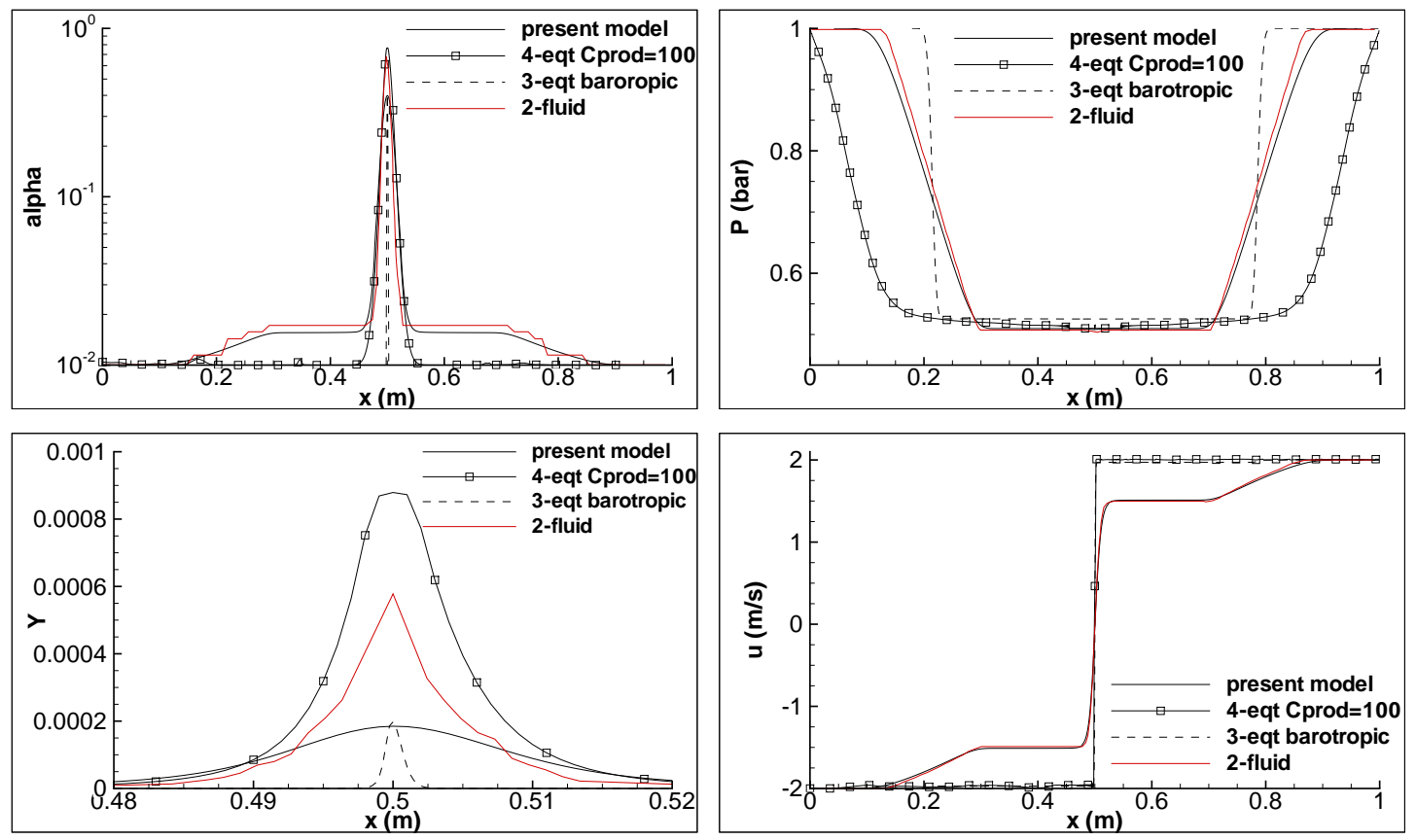

Figure 9: Water-gas double rarefaction with cavitation $\left|u_{0}\right|=2 \mathrm{~m} / \mathrm{s}$, models comparison, mesh 1000 cells, $t=3.2$ ms. Void ratio, pressure and velocity profiles along the tube, enlargement of the mass fraction between abscissa $0.48-0.52 \mathrm{~m}$.

First, we propose a comparison of the considered numerical schemes. As previously, VF Roe, HLLC v1 and AUSM+up schemes were not able to provide a solution. The evolution of mass and volume fractions, pressure and velocity are plotted in Fig. 10. The two evaporation fronts are clearly highlighted. The decrease of pressure in the cavitation pocket, due to the temperature cooling associated to the evaporation phenomenon, is well simulated. The intensity is more pronounced using the Jameson scheme. In comparison with the 2-fluid solution, evaporation fronts are stiffer. The void ratio profiles show that the cavitation pocket is quasi fill of pure vapor. As previously, discrepancies are noticeable for the maximum value of the mass fraction of gas, but with smaller gaps. The better agreement is obtained with the Jameson scheme and the HLLC 30 provides the highest value ( $25 \%$ of error). Using the Jameson scheme, we clearly observe oscillations of the solution close to the evaporation fronts.

We compare now the present model with the barotropic formulation and the Kunztype model. For the barotropic model, it was not possible to obtain a solution (we did not try to modify the value of parameter $c_{\text {baro }}$ ). For the Kunz-type model, the calibration of $C_{\text {prod }}$ was performed in order to fit the reference solution of Zein et al. [40] for the void ratio. The closest result was obtained with $C_{\text {prod }}=10^{5} \mathrm{~m}^{3} / \mathrm{kg}$, that is a factor $1000 \mathrm{in}$ comparison with the previous case. The large variation of the parameter $C_{\text {prod }}$ is clearly questionable. 

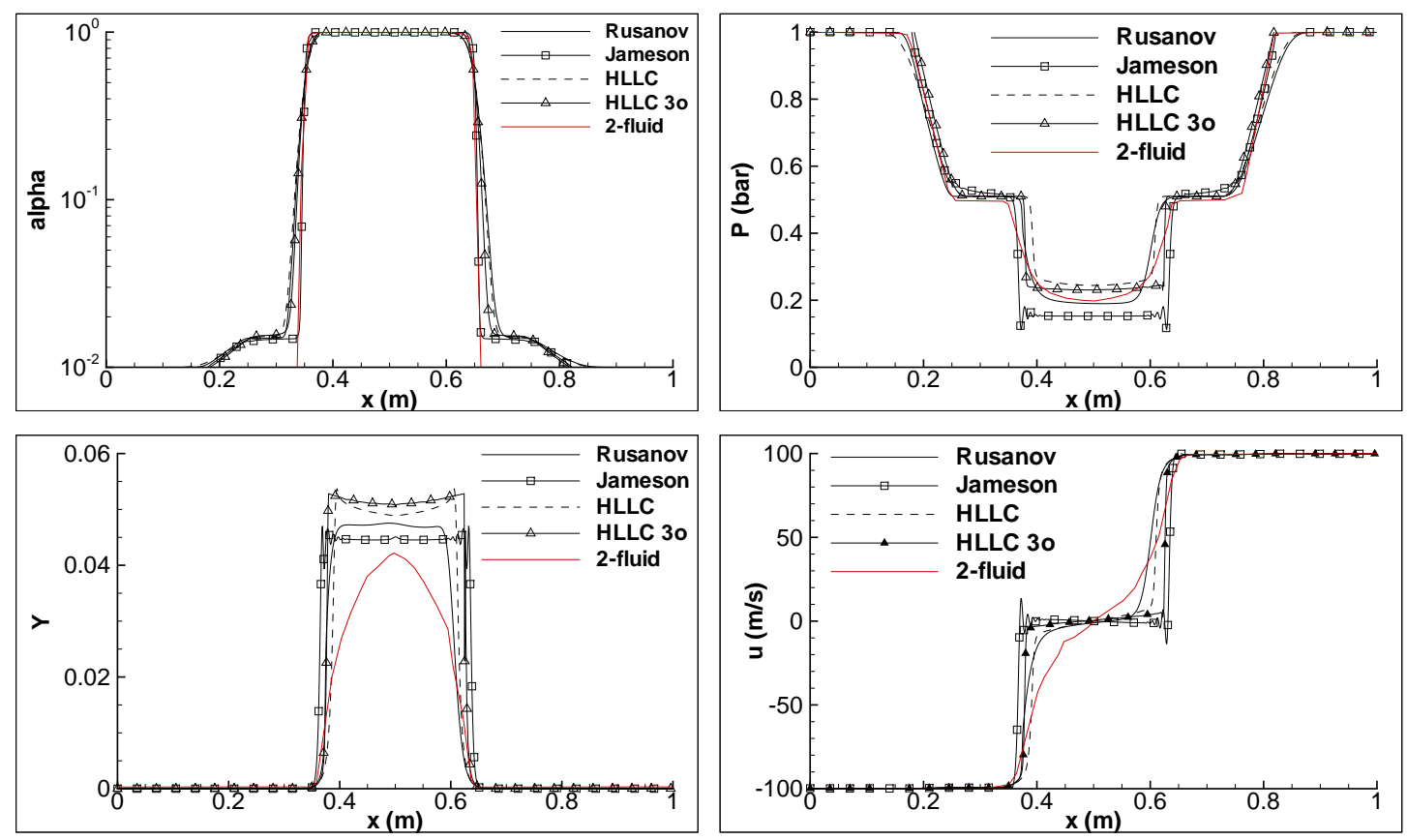

Figure 10: Water-gas double rarefaction with cavitation $\left|u_{0}\right|=100 \mathrm{~m} / \mathrm{s}$, numerical schemes comparison, mesh 1000 cells, $t=1.5 \mathrm{~ms}$. Void ratio, pressure, mass fraction and velocity profiles.

The evolution of mass and volume fractions, pressure and velocity are plotted in Fig. 11. As observed for the previous case, the Kunz-type model provides a cavitation pocket with good values of void ratio. Yet, the mass fraction is over-estimated by a factor 4 . For the pressure distribution, the rarefaction waves are badly simulated and the evaporation fronts are not visible. The velocity profile illustrates oscillations of the solution near positions of evaporation fronts.

To conclude, both double rarefaction test cases with cavitation show the ability of the present model and the numerical treatment to correctly simulate the cavitation pocket development and all wave fronts. On the contrary, the 4-equation Kunz-type formulation fails to compute these cases. It is certainly due to its lack of thermodynamic coherence. Moreover, a key point in the void ratio formulation is the term $\operatorname{Kdiv}(\vec{V})$, which takes into account the effects of changes in volume of each phase. This term is missing in the Kunz-type model.

\section{Computational results on two-dimensional two-phase problems}

In this section, we present simulation results for two-dimensional problems: a shockinduced bubble collapse and an underwater explosion. Due to difficulties discussed pre- 

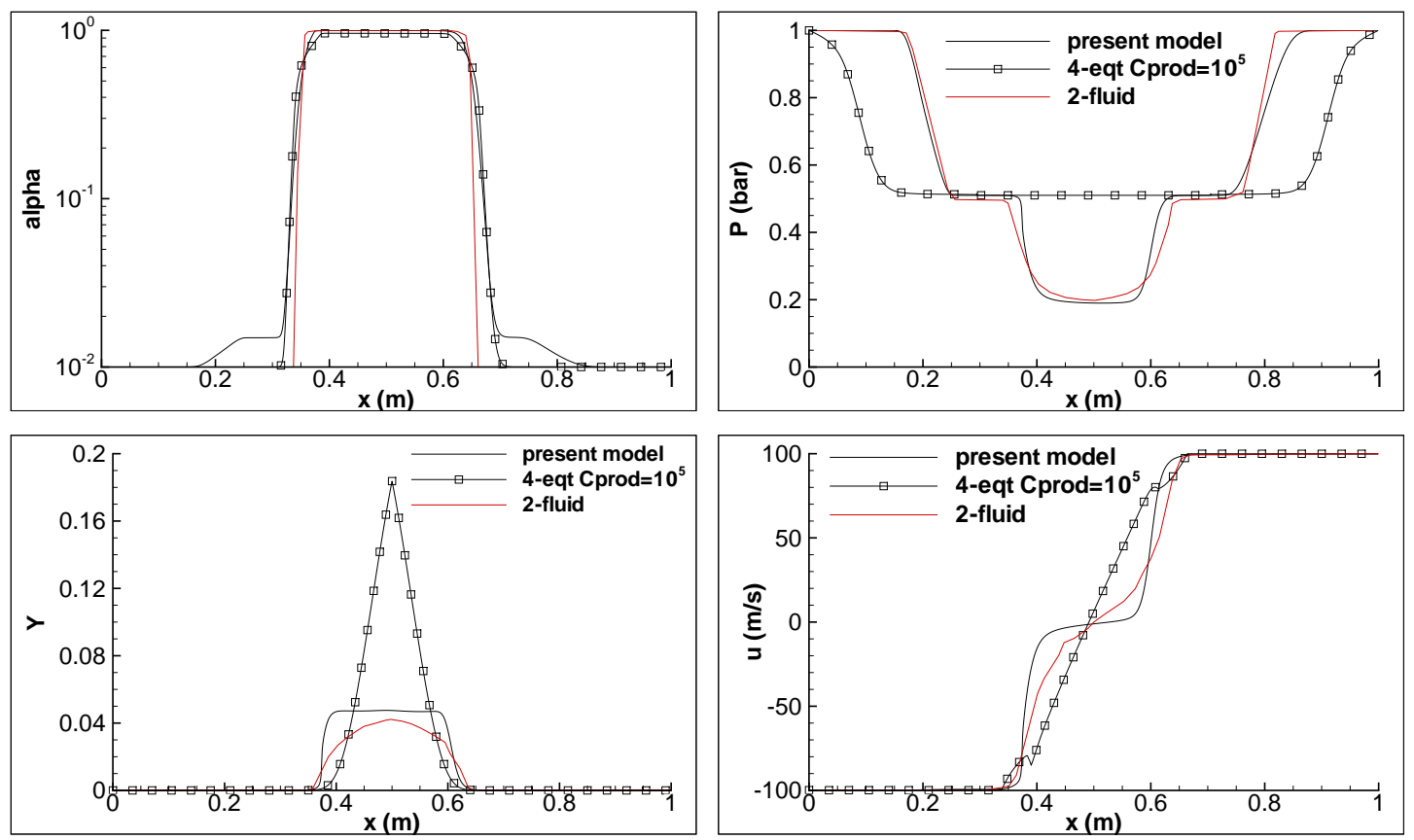

Figure 11: Water-gas double rarefaction with cavitation $\left|u_{0}\right|=100 \mathrm{~m} / \mathrm{s}$, models comparison, mesh 1000 cells, $t=1.5 \mathrm{~ms}$. Void ratio, pressure, mass fraction and velocity profiles.

viously on one-dimensional cases, VF Roe, HLLC v1 and AUSM-type schemes will be not considered in the following.

\subsection{Shock-bubble interaction}

The considered test is similar to the one presented in $[42,43]$. A cylindrical air bubble, $6 \mathrm{~mm}$ in diameter, is immersed in a water pool, under the following initial conditions: $\vec{V}=(0,0) \mathrm{m} / \mathrm{s}, P=10^{5} \mathrm{~Pa}, \rho_{\text {air }}=1 \mathrm{~kg} / \mathrm{m}^{3}$ and $\rho_{\text {water }}=1000 \mathrm{~kg} / \mathrm{m}^{3}$. The center of the bubble is located at $(9,6) \mathrm{mm}$ in the computational domain of size $12 \times 24 \mathrm{~mm}$. The bubble is collapsed by a normal shock wave moving at $M_{s h}=1.72$, initially located at abscissa $x_{s h}=4 \mathrm{~mm}$. Parameters of the EOSs and post-shock conditions are:

$$
\begin{aligned}
& \left(\begin{array}{c}
\gamma \\
P_{\infty} \\
\rho
\end{array}\right)_{\text {Liquid }}=\left(\begin{array}{c}
4.4 \\
6 \times 10^{8} \mathrm{~Pa} \\
1000 \mathrm{~kg} / \mathrm{m}^{3}
\end{array}\right) ; \quad\left(\begin{array}{c}
\gamma \\
P_{\infty} \\
\rho
\end{array}\right)_{\mathrm{Gas}}=\left(\begin{array}{c}
1.4 \\
0 \mathrm{~Pa} \\
1 \mathrm{~kg} / \mathrm{m}^{3}
\end{array}\right), \\
& \left(\begin{array}{c}
\mathrm{P} \\
\rho \\
\mathrm{u}
\end{array}\right)_{\text {post-shock }}=\left(\begin{array}{c}
1.910^{9} \mathrm{~Pa} \\
1323.65 \mathrm{~kg} / \mathrm{m}^{3} \\
681.58 \mathrm{~m} / \mathrm{s}
\end{array}\right) .
\end{aligned}
$$

The top and bottom boundary conditions are assumed to be walls, and the boundary conditions for the left and right sides are assumed to be non-reflecting. The schematic 


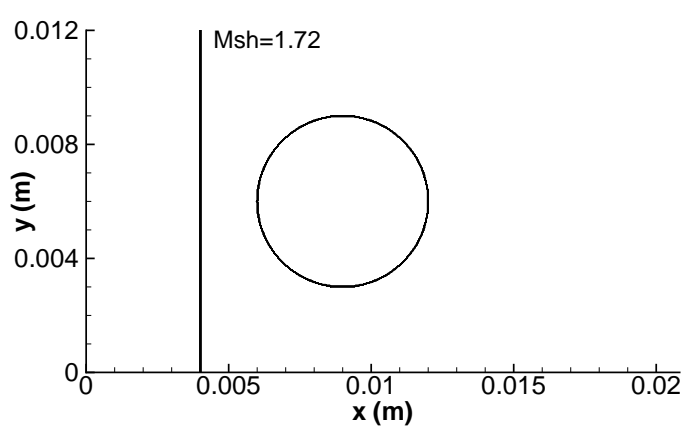

Figure 12: Initial situation for the shock-bubble interaction.

diagram of the test case is given in Fig. 12. Simulations are performed using an uniform grid composed by $800 \times 400$ cells and a time step $\Delta t=10^{-9} \mathrm{~s}$. For all simulations, a very weak volume fraction of gas $\alpha_{0}=10^{-10}$ is added to the liquid phase.

Firstly, a description of the main phenomena involved in this shock-bubble interaction is proposed based on the second-order HLLC simulation. Note that the mass transfer term is not activated in this study. The time evolution of the density gradient modulus (Schlieren-type representation) is plotted in Fig. 13. After the water shock wave has collided with the bubble, at time $t=2 \mu \mathrm{s}$, a strong rarefaction wave is reflected backwards from the interface and a weak shock wave transmits from inside of the bubble.

At time $t=3 \mu \mathrm{s}$ the incident water shock has traversed almost the full cavity width. The interaction between this shock and the expansion waves originating at the bubble surface has resulted in significant weakening and curvature of the shock. The shock inside the bubble propagates more slowly. Due to the pressure difference between back and forth of pressure waves, the bubble is asymmetrically contracted with concave shape. As time moves on, the bubble becomes kidney shaped and spreads laterally in the process. This change in shape is driven by vorticity generated at the edge of the bubble due to the passage of the wave which induces a jet of water along the axis of flow symmetry.

When this jet impinges on the water at the downstream edge of the bubble, at time $t=3.6 \mu \mathrm{s}$, the bubble forms a pair of distinct vortical structures.

Such physical phenomena were described in the case of shock-bubble interactions. According to the sign of the difference of acoustic impedance of pure phases $d R=\rho_{v} c_{v}-$ $\rho_{l} c_{l}$, two scenarios are depicted. In our case, $d R<0$ and this situation is commonly referred to as divergent geometry [44]. After several shock-passage times, the features observed in the flow field are dominated by the vortical motion.

On impact with the bubble, the jet produces an intense blast wave (also called water hammer shock [45]) in the surrounding water, which is clearly illustrated after time $t=3.6$ $\mu s$. The developing blast wave advances relatively slowly to the left below the bubble, due to the high water velocity in the jet fluid, so that the blast front is highly asymmetric. The rightward blast wave increases as a spherical wave. Both shocks lose strength as they advance, the rightward wave more so than its leftward twin. 
a)

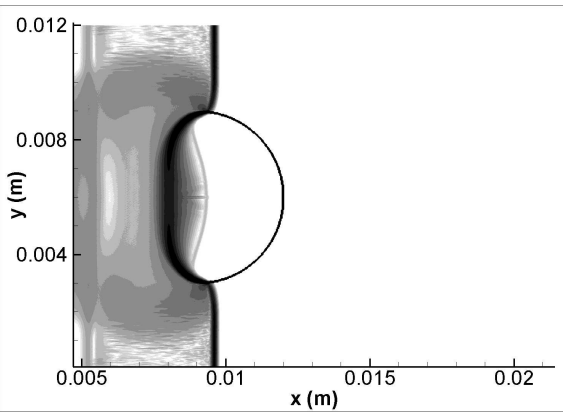

b)

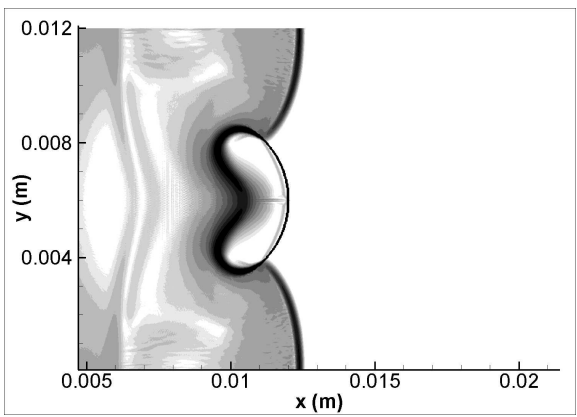

C)

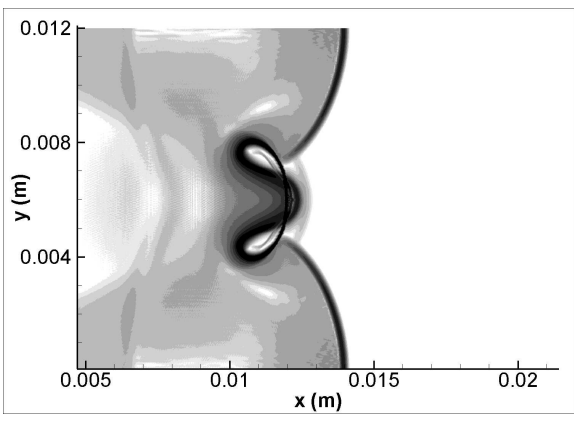

d)

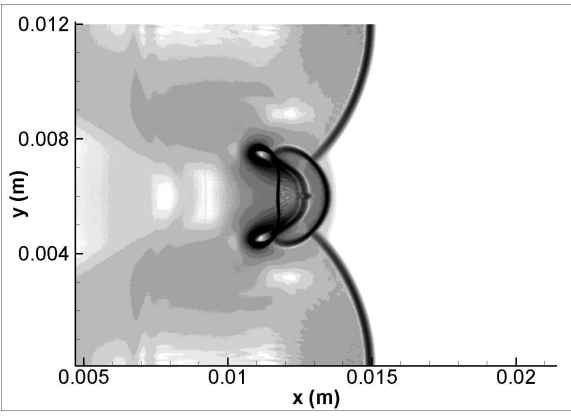

e)

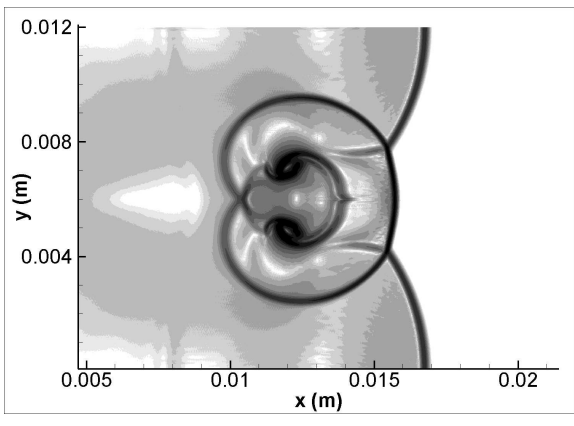

f)

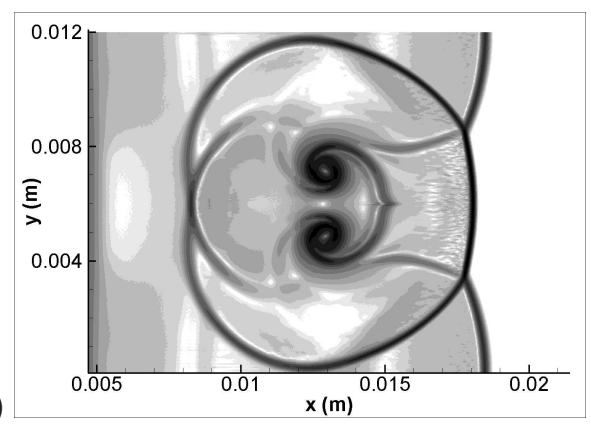

Figure 13: Shock-bubble interaction, evolution of the density gradient at times: a) $t=2 \mu \mathrm{s}, \mathrm{b}) t=3 \mu \mathrm{s}, \mathrm{c})$ $t=3.6 \mu \mathrm{s}, \mathrm{d}) t=4 \mu \mathrm{s}, \mathrm{e}) t=4.8 \mu \mathrm{s}$, and f) $t=5.6 \mu \mathrm{s}$. HLLC 2 nd order scheme, mesh $800 \times 400$ cells.

Caused by the leftward blast wave, secondary jets penetrate into the smaller bubbles and cut the initial bubble into four pieces (time $t=4.8 \mu \mathrm{s}$ ). The interaction of the blast wave with the remains of bubble generates high pressure levels [46]. Finally, the blast wave continues its expansion and the cavity its shrinkage.

The pressure evolution is illustrated in Fig. 14. During the impact of the water jet with the stationary water at the front of the bubble, a high-pressure zone is formed and a blast wave is generated (see time $t=3.6 \mu \mathrm{s}$ ). This blast wave expands continuously in the radial direction. At time $t=4.8 \mu \mathrm{s}$, the low-pressure area inside the vortices core are well illustrated. The interaction of the leftward blast wave with the cavity leads to a very strong pressure peak, which is the most intense during the collapse. The pressure 
a)

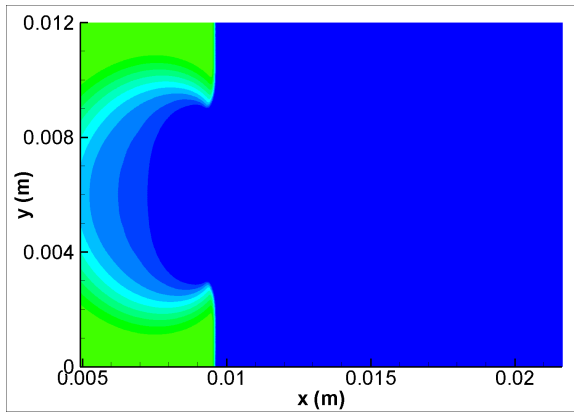

b)

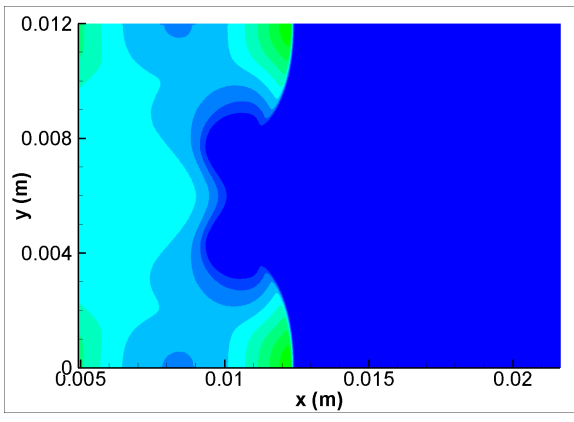

c)

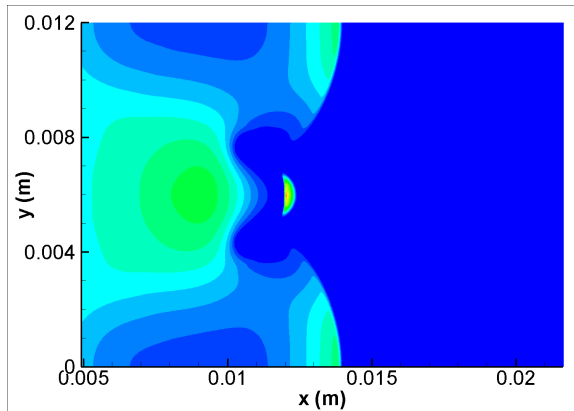

d)

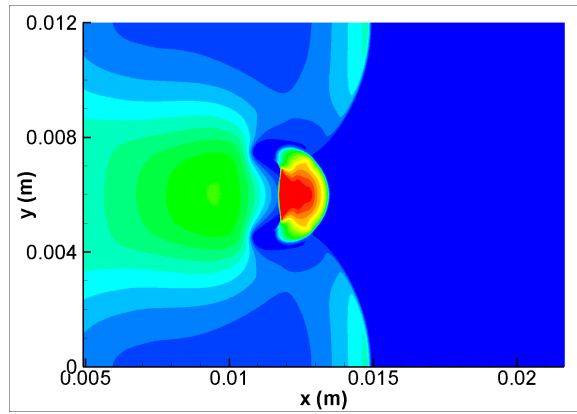

e)
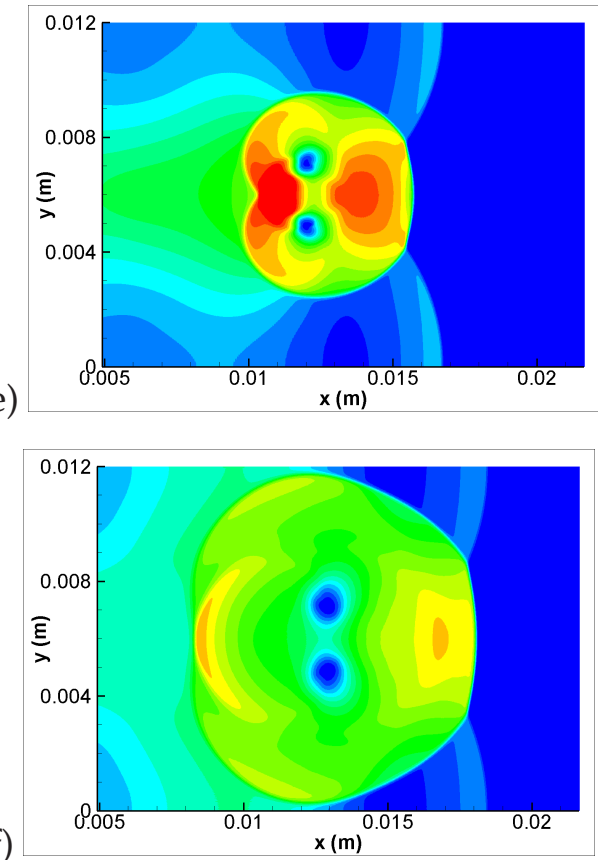

Figure 14: Shock-bubble interaction, evolution of the pressure (in bar) at times: a) $t=2 \mu s, \mathrm{~b}) t=3 \mu s, \mathrm{c}$ ) $t=3.6 \mu \mathrm{s}, \mathrm{d}) t=4 \mu \mathrm{s}$, e) $t=4.8 \mu \mathrm{s}$, and f) $t=5.6 \mu \mathrm{s}$. HLLC 2 nd order scheme, mesh $800 \times 400$ cells.

amplitude depends on the initial bubble size and the incident shock intensity. These results show that the most phenomenon associated to this interaction such as the water jet formation, the bubble division, the formation of a high-pressure zone and the generation of a strong blast wave shock which expands in the liquid are well captured in comparison with previous numerical results $[42,43,46]$.

Secondly, the influence of the numerical scheme is investigated. The dissipation coefficients for the Jameson scheme are $\left(k^{(2)}, k^{(4)}\right)=(1.5,0.1)$. The use of smaller values for 
a)

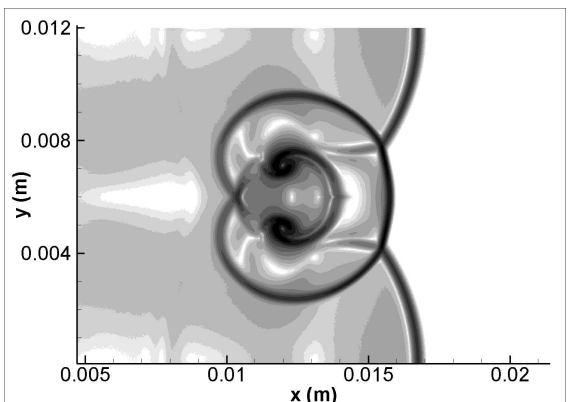

b)

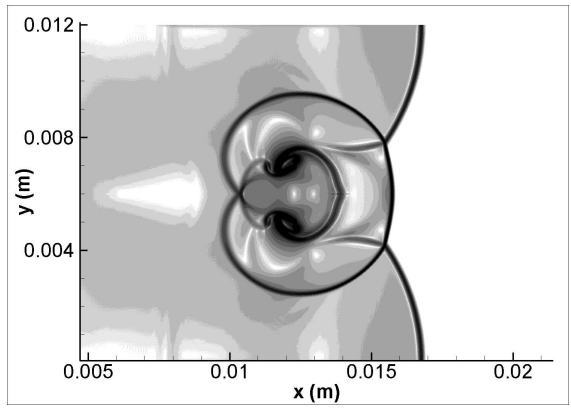

c)

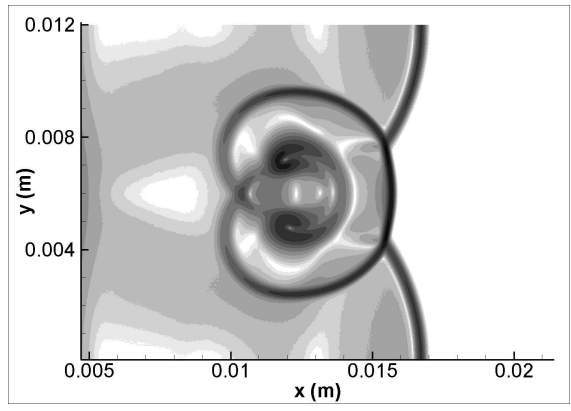

d)

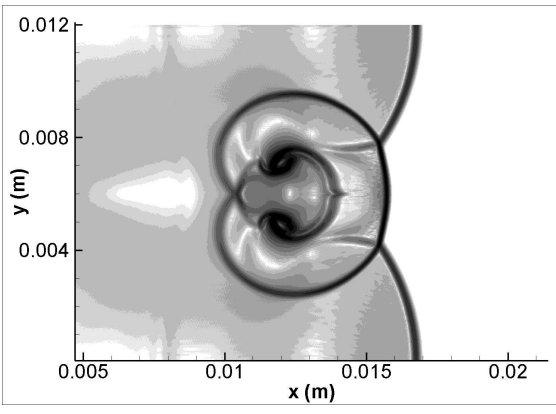

e)

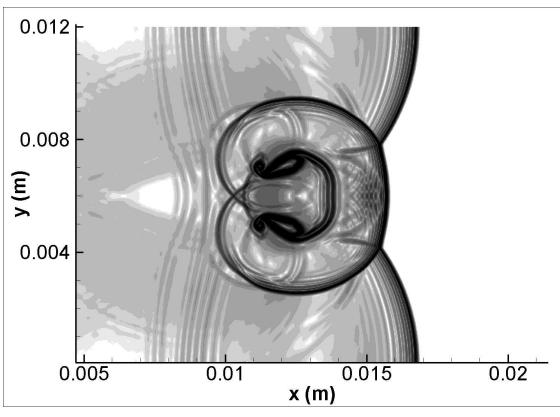

f)

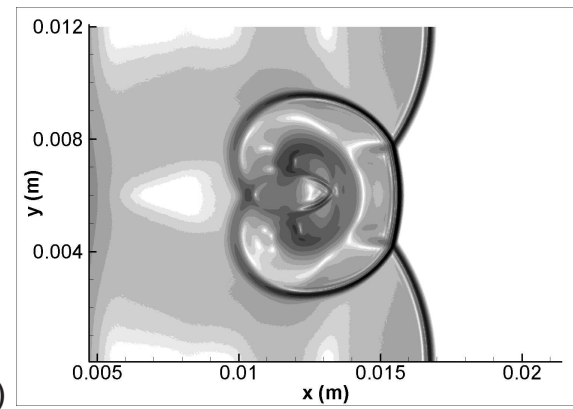

Figure 15: Shock-bubble interaction, comparison of the density gradient contour at time $t=4.8 \mu$ s obtained with different schemes, mesh $800 \times 400$ cells: a) HLLC, b) HLLC finer mesh $1600 \times 800$ cells, c) Rusanov, d) HLLC 2nd-order, e) Jameson and f) LD-Rusanov.

the parameter $k^{(4)}$ led to large oscillations of the solution. Moreover, it was not possible to compute the case using the third-order HLLC scheme and also the second-order LD-Rusanov scheme.

The density gradient modulus obtained with all schemes is plotted in Fig. 15 at time $t=4.8 \mu \mathrm{s}$. As regard to the cavity shape and the presence of four pieces of the initial bubble, this phenomenon is clearly highlighted using the Jameson scheme, Fig. 15e. Yet, spurious oscillations are generated near discontinuities. The bubble cutting phenomenon is not captured by the HLLC scheme (Fig. 15a) and only a little piece can be observed with the second-order formulation, Fig. 15d. Using a finer grid composed by $1600 \times 800$ cells, the four pieces are well simulated with the HLLC scheme, Fig. 15b. As expected, 
a)

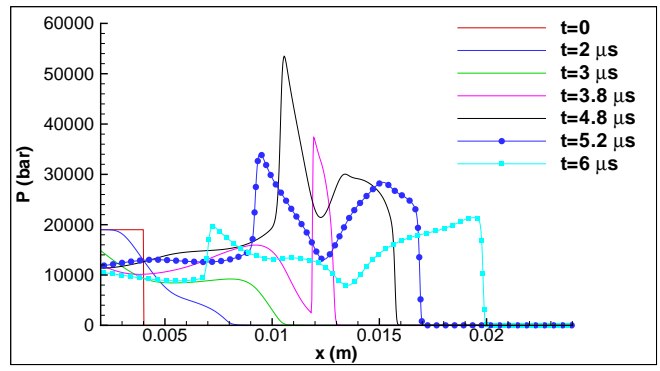

b)

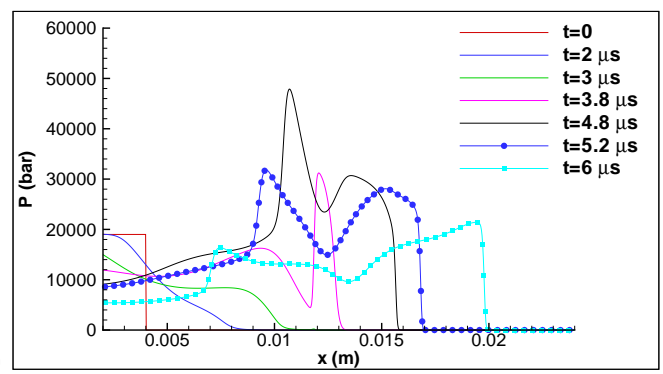

C)

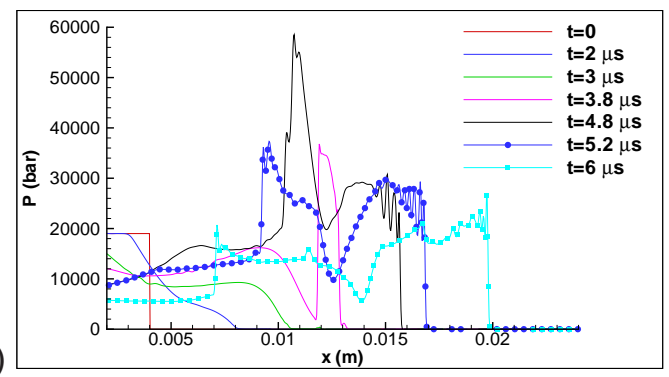

d)

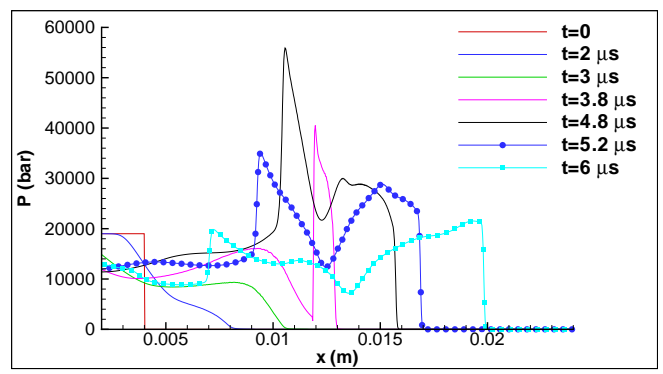

e)
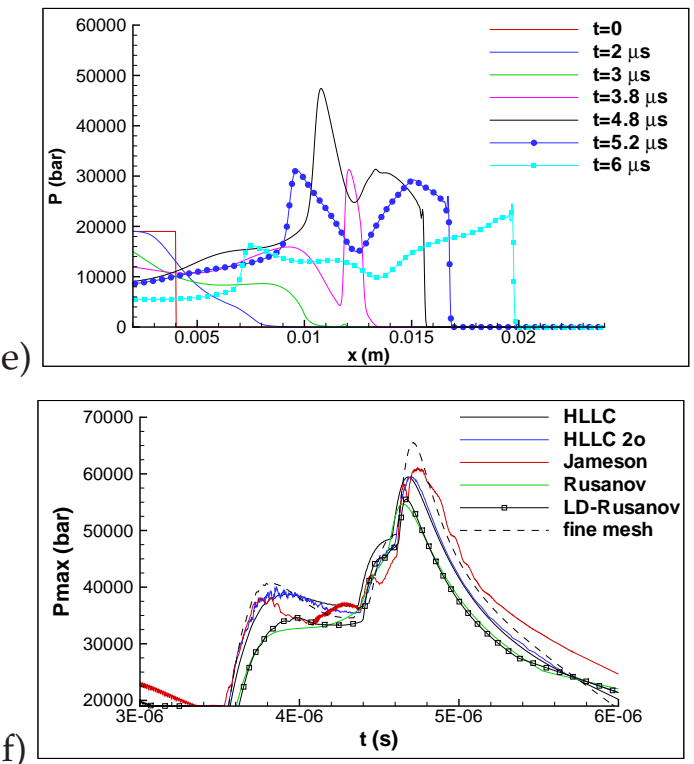

Figure 16: Shock-bubble interaction, evolution of the pressure during the bubble collapse on the symmetry axis $y=6 \mathrm{~mm}$, numerical schemes comparison, mesh $800 \times 400$ cells: a) HLLC, b) Rusanov, c) Jameson, d) HLLC 2nd order and e) LD-Rusanov. In f) evolution of the maximum pressure during the collapse.

the numerical dissipation of the Rusanov scheme is huge, Fig. 15c. The low-diffusive formulation improves the fronts capturing but not the bubble shape evolution, Fig. $15 \mathrm{f}$.

The pressure evolution in a cutting plane $y=6 \mathrm{~mm}$ (corresponding to the symmetry axis) is plotted in Fig. 16 for all schemes and highlights the pressure peaks during the cavity collapse. For all simulations, we can observed the first peak at time $t=3.8 \mu s$ when the water jet impacts the bubble front and the second peak (more intense) at time $t=4.8 \mu \mathrm{s}$ when the leftward blast wave collides the remains of bubble. The intensity of both peaks varies with the scheme. The evolution of the maximum pressure during the collapse is plotted in Fig. 16f. The simulation performed with the finer grid, $1600 \times 800$ cells, predicts a first peak at time $3.8 \mu \mathrm{s}$ around 40000 bar (the double of the incident shock wave intensity) and the second peak at time $4.7 \mu$ s around 65000 bar. The lowest amplitude, around 55000 bar, is provided by the Rusanov scheme. The use of the low-diffusive formulation slightly improves both peaks. The peak predicted by the HLLC scheme is around 60000 
bar and the effect of the second-order formulation is very weak. The solution obtained with the Jameson scheme presents two consecutive peaks at times 4.65 and $4.75 \mu s$ with an amplitude around 60000 bar. In comparison with previous simulations, we obtain an intermediate value between results of Ball et al. - 4.7 GPa [42] - and Nourgaliev et al. 10.1 GPa [43].

\subsection{Underwater explosion with cavitation}

The cylindrical underwater explosion near a flat free surface is considered. A similar case has been studied in $[47,48]$. The initial conditions are given as follows. A high-pressured gas cylinder with a radius of $0.5 \mathrm{~m}$ is located in the center of the computational domain and the initial flow parameters for explosive gas, water and air are:

$$
\begin{aligned}
& \left(\begin{array}{l}
\gamma_{g} \\
\rho_{g} \\
P_{g} \\
V_{g}
\end{array}\right)_{\text {Gas }}=\left(\begin{array}{c}
2 \\
1270 \mathrm{~kg} / \mathrm{m}^{3} \\
8290 \mathrm{bar} \\
0
\end{array}\right) ; \quad\left(\begin{array}{l}
\gamma_{l} \\
\rho_{l} \\
P_{l} \\
V_{l}
\end{array}\right)_{\text {Liquid }}=\left(\begin{array}{c}
2.35 \\
1150 \mathrm{~kg} / \mathrm{m}^{3} \\
1 \mathrm{bar} \\
0
\end{array}\right), \\
& \left(\begin{array}{c}
\gamma_{\text {air }} \\
\rho_{\text {air }} \\
P_{\text {air }} \\
V_{\text {air }}
\end{array}\right)_{\text {Air }}=\left(\begin{array}{c}
1.4 \\
1 \mathrm{~kg} / \mathrm{m}^{3} \\
1 \mathrm{bar} \\
0
\end{array}\right) .
\end{aligned}
$$

The stiffened gas parameters for water and its vapor are similar to the previous double rarefaction case with cavitation. The vapor pressure is $P_{\text {vap }}=51000 \mathrm{~Pa}$. The quantity $d P / d T$ is set to a low value, i.e. $100 \mathrm{~Pa} / \mathrm{K}$. The computational domain is a rectangular region with $x \times y \in[0,12] \times[0,12]$ and the free surface is located at the straight line $y=8$ $\mathrm{m}$ (see Fig. 17). An uniform grid composed by $400 \times 400$ cells is distributed in computational domain. The top and bottom boundary conditions are walls. On other frontiers,

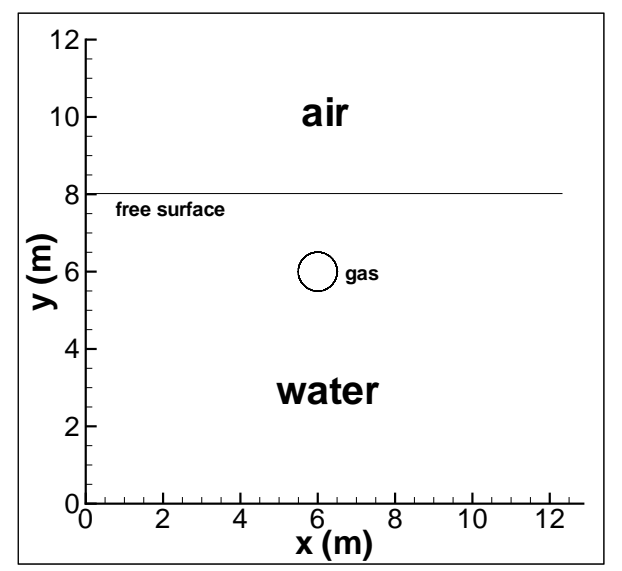

Figure 17: Initial condition for the underwater explosion. 
a)

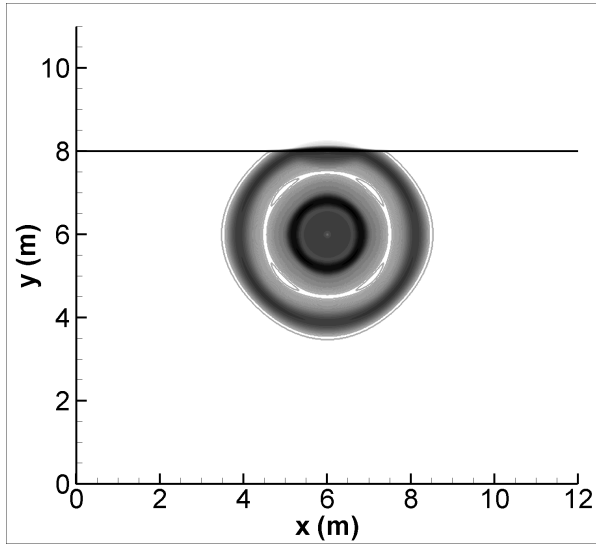

b)

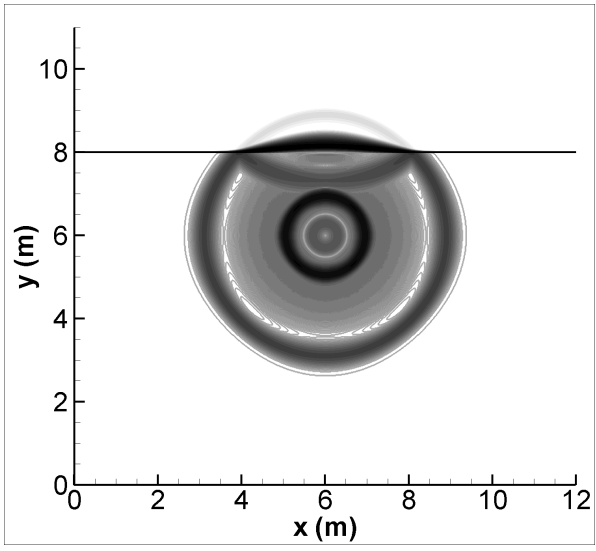

C)

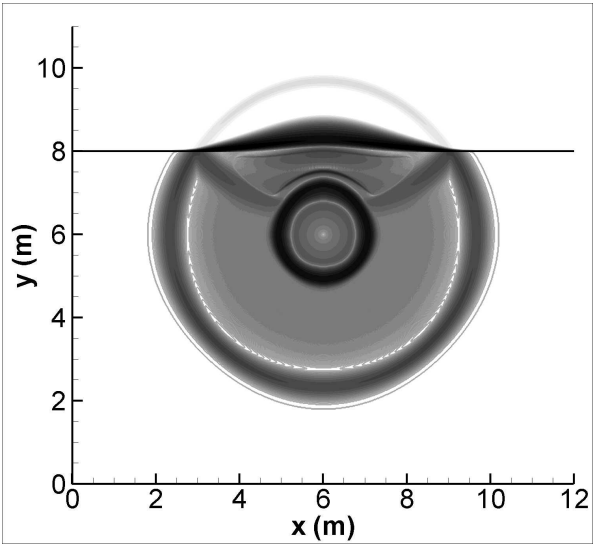

d)

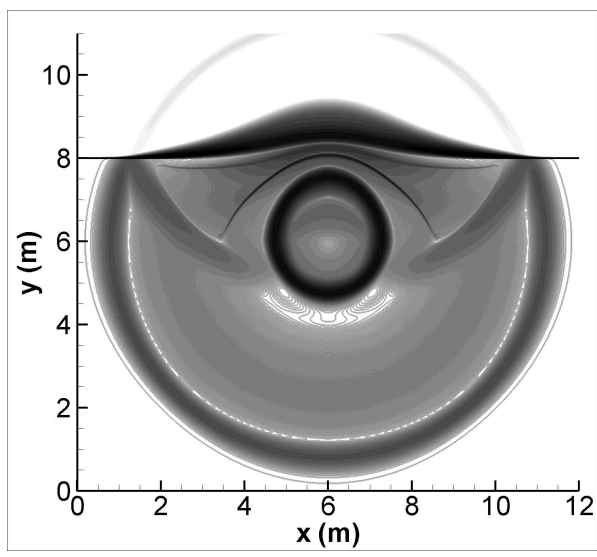

Figure 18: Underwater explosion, evolution of the density gradient at times: a) $t=1 \mathrm{~ms}, \mathrm{~b}) t=1.5 \mathrm{~ms}, \mathrm{c}) t=2$ ms, d) $t=3 \mathrm{~ms}$. HLLC 2 nd order scheme, mesh $400 \times 400$ cells.

variables are extrapolated. The time step is set to $10^{-6} \mathrm{~s}$. As previously, a very weak volume fraction of gas $\alpha_{0}=10^{-10}$ is added to the liquid phase.

A first simulation is performed using the second-order HLLC scheme in order to describe the main physical phenomena. Fig. 18 illustrates a series of density gradient contours as time progresses where the shock and free surface interaction are clearly observed. At $t=1.0 \mathrm{~ms}$, Fig. 18a, the initial shock generated by the explosion impacts the free surface. It reflects with strong rarefaction waves while a transmitted shock is propagating into the air medium, Fig. 18b. The reflected rarefaction wave interacts with the explosion zone's interface, leading to a formation of the reflected compression wave and a transmitted rarefaction wave, Fig. 18c. Next, due to the interaction between the reflected rarefaction wave and upper boundary of the bubble, the top section of the bubble is accelerated in the vertical direction, causing an elongation of the bubble shape, Fig. 18d.

The density evolution is plotted in Fig. 19 and illustrates the initial shock wave propagation, its impact with the free surface, the generation of the reflected rarefaction wave 
a)

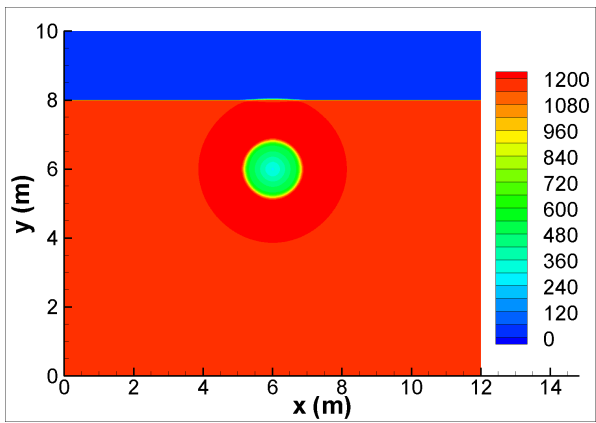

b)

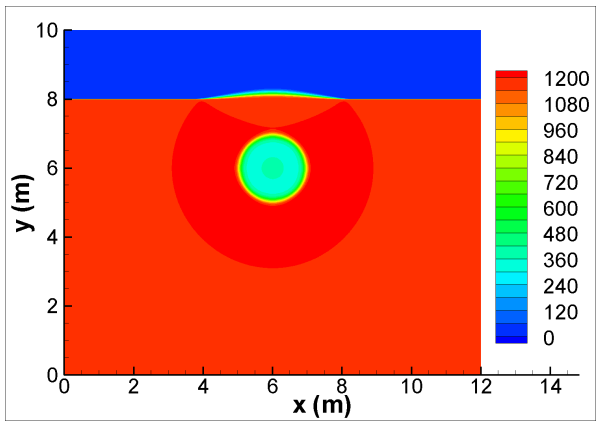

C)

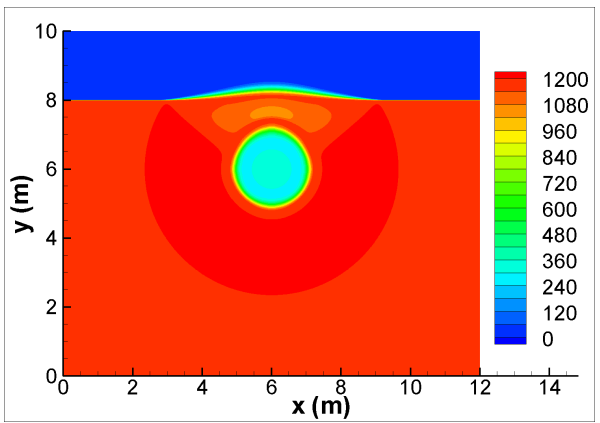

d)

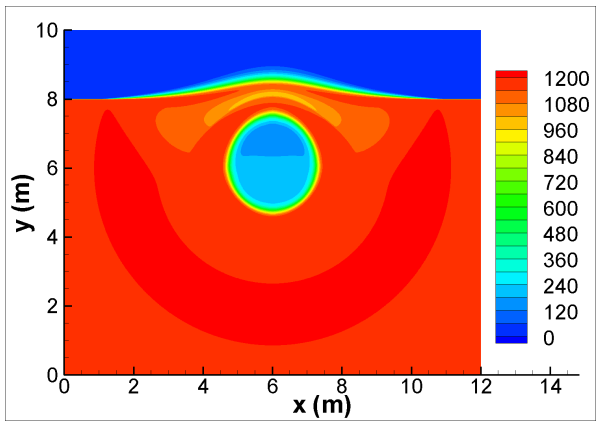

Figure 19: Underwater explosion, evolution of the density $\left(\mathrm{kg} / \mathrm{m}^{3}\right)$ at times: a) $\left.\left.t=1 \mathrm{~ms}, \mathrm{~b}\right) t=1.5 \mathrm{~ms}, \mathrm{c}\right) t=2$ $\mathrm{ms}, \mathrm{d}) t=3 \mathrm{~ms}$. HLLC 2 nd order scheme, mesh $400 \times 400$ cells.

and its interaction with the gas area leading to the elongated bubble shape. At the same time, the free surface is disturbed and moves upwards due to relief of the main shock.

A numerical schemes comparison is proposed, especially as regard to the cavitation pocket development. The dissipation parameters for the Jameson scheme are $\left(k^{(2)}, k^{(4)}\right)=$ $(1.5,0.085)$. Note that the second-order HLLC scheme is started from a first-order solution on 1000 iterations. The starting from the initial condition led to the computation divergence.

Isolines of pressure at time $t=3 \mathrm{~ms}$ are presented in Fig. 20 for all schemes. The isoline at the vapour pressure value (i.e. $51000 \mathrm{~Pa}$ ) is plotted in order to observe the cavitation evolution. The rarefaction waves generated by the impact of the initial shock wave with the free surface cause the water pressure just below the free surface to drop very rapidly. With the decrease of the water pressure, a cavitation pocket appears just below the free surface. All simulations predict the appearance and growth of such cavitation pocket. The size of the pocket varies with the numerical scheme. The simulation performed on a finer grid, $800 \times 800$ cells, with the HLLC scheme highlights the shape of the cavitation pocket, Fig. 20b. Except the Jameson simulation, Fig. 20e, all results under-estimate the cavitation development and predict two separated pockets. Yet, as observed previously, the Jameson solution provides spurious oscillations. The second-order HLLC scheme improves the cavitation prediction in comparison with the first-order result by predicting 
a)
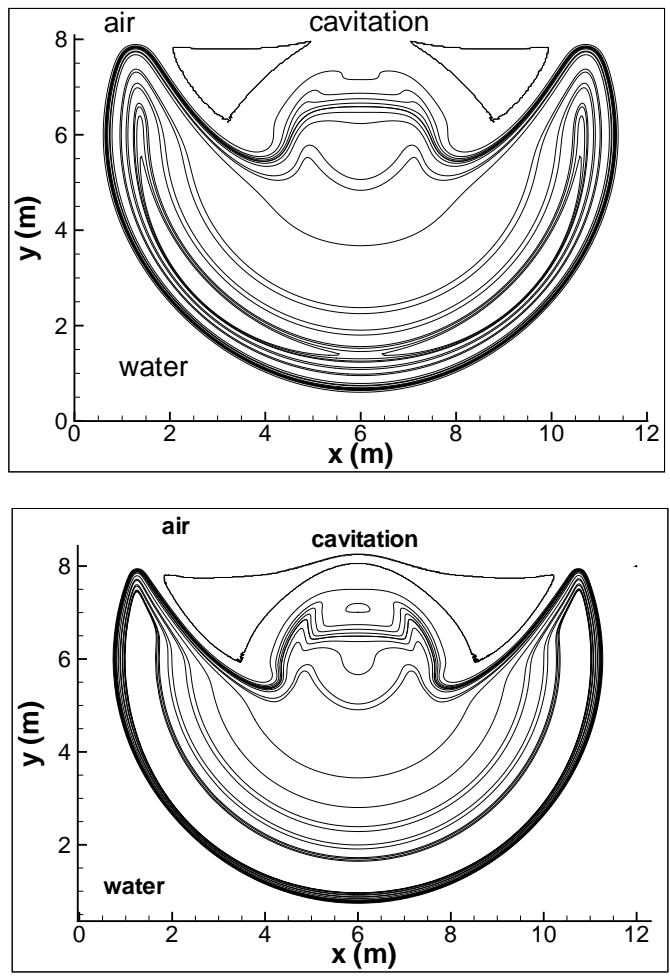

b)

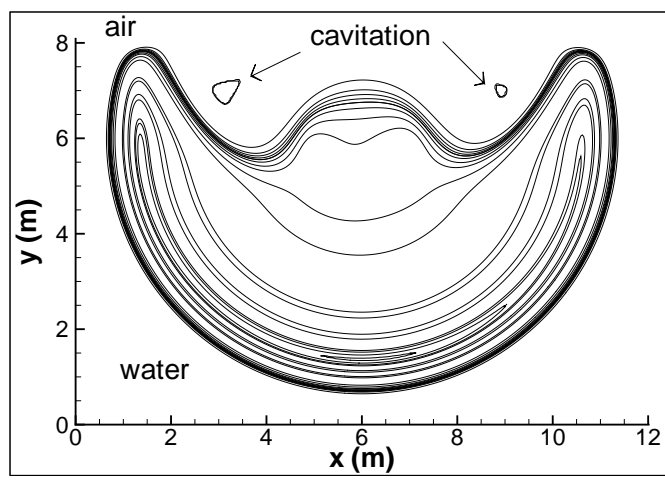

d)

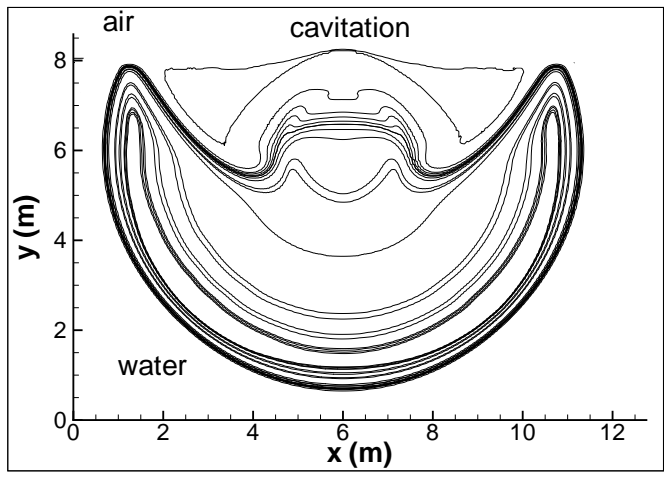

e)
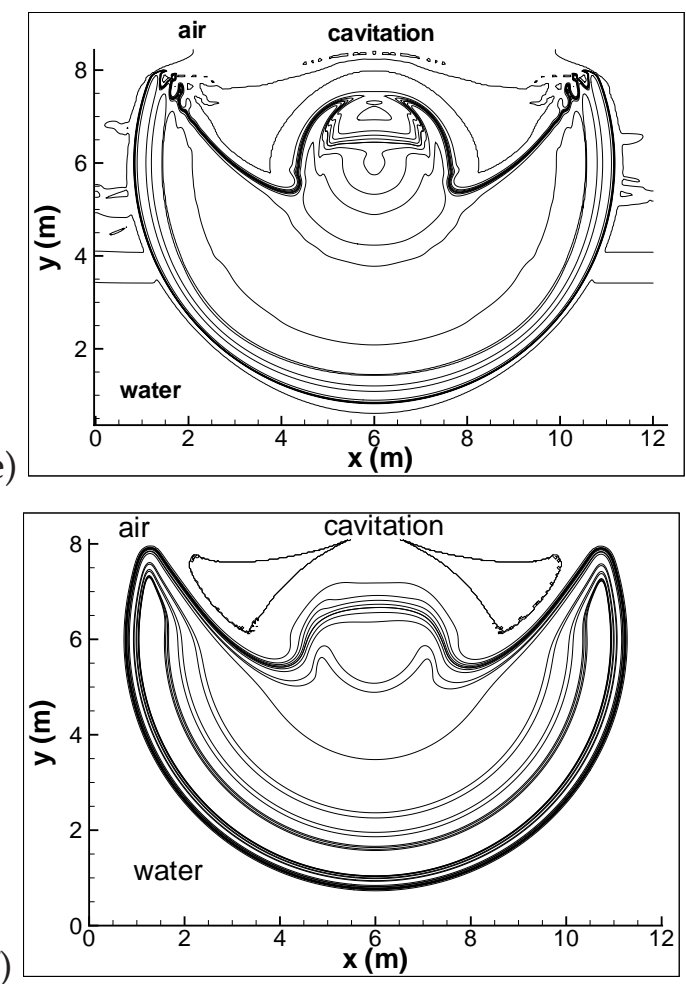

Figure 20: Underwater explosion, isoline of pressure and cavitation pocket at time $t=3 \mathrm{~ms}$, numerical schemes comparison, mesh $400 \times 400$ cells: a) HLLC, b) HLLC finer mesh $800 \times 800$ cells, c) Rusanov, d) HLLC 2nd-order, e) Jameson and f) LD-Rusanov.

a quasi single pocket, Fig. 20d. The two cavitation pockets obtained with the Rusanov simulation are very small (Fig. 20c) and the use of the low-diffusive formulation largely increases the pockets size (Fig. 20f).

Finally, the pressure profile at time $t=3 \mathrm{~ms}$ in a cutting plane $x=3 \mathrm{~m}$ is plotted in Fig. 21 for all schemes. It illustrates the shock wave front $(y \simeq 2 \mathrm{~m})$ and the explosion area, the rarefaction front $(y \simeq 6 \mathrm{~m})$ generating the cavitation pocket, the free surface area 


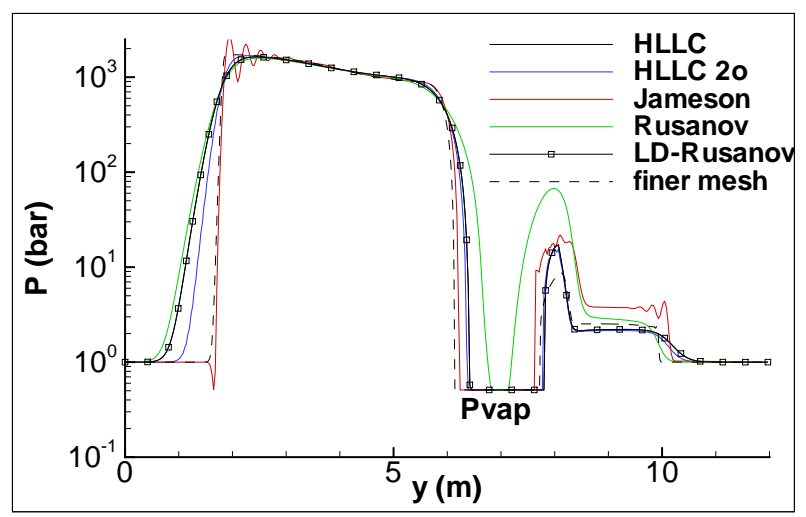

Figure 21: Underwater explosion, pressure profile at time $t=3 \mathrm{~ms}$ in a cutting plane $x=3 \mathrm{~m}$, numerical schemes comparison, mesh $400 \times 400$ cells.

$(y \simeq 8 \mathrm{~m})$ and the transmitted shock wave front in air $(y \simeq 10 \mathrm{~m})$. As regard to the shock wave front, the Jameson solution is in close agreement with the finer mesh result. Yet, oscillations are noticeable even using large values of the artificial dissipation coefficient. With other schemes, the shock front is spread. The second-order MUSCL formulation improves a little the solution in comparison with the first-order HLLC scheme. Concerning the cavitation pocket extension, all results are quite similar, except using the Rusanov scheme. The low-diffusive formulation largely improves the cavitation development, as observed previously. The transmitted shock wave front in air is spread, except using the Jameson scheme. Yet, the post-shock value is over-estimated with the Jameson scheme in comparison with other results and with the finer mesh solution.

\section{Conclusions}

A void ratio transport-equation model involving mass transfer was presented and implemented in a one-fluid two-phase compressible software. Different numerical schemes were proposed to integrate the system based on a finite volume approach: HLLC, Rusanov, VF Roe, Jameson-Schmidt-Turkel and AUSM-type schemes. The behavior of the numerical tool was studied and verified by several benchmark problems. First, the computation of one-dimensional shock tube problems demonstrated the ability of the proposed model and schemes to capture strong shock waves adequately by comparison with analytical solution or two-fluid solution. Secondly, double rarefaction cases leading to the apparition of cavitation were simulated. These cases illustrated difficulties for Riemann solver to capture strong rarefaction and evaporation fronts. It was not possible to compute accurately these cases using both VF Roe and AUSM-type schemes. Moreover, the importance of the numerical treatment for the void ratio equation was highlighted using the HLLC scheme. The non-conservative term has to be carefully discretised to obtain a good solution. 
Then, a two-dimensional shock-bubble interaction leading to the bubble collapse was simulated. This case demonstrated the ability of the model associated to the HLLC scheme to capture the main physical phenomena: the blast wave development and the interaction with the bubble pieces leading to the largest pressure peak. It showed also the robustness of the present solver in the multidimensional framework. The influence of the numerical schemes was analyzed on an intermediate refined grid. As regard to the bubble cutting during the collapse, only the second-order schemes (HLLC-MUSCL and Jameson) predicted this phenomenon. The low-diffusive formulation for the Rusanov scheme did not clearly improve the solution.

Finally, the numerical tool was applied to an underwater explosion near a free surface for which a cavitation pocket develops. Results clearly showed that the model associated to the HLLC scheme is well-suited to simulate the formation and development of cavitation zones in a complex compressible flow. As regard to the cavitation pocket prediction, the low-diffusive LD-Rusanov formulation largely improved the solution. Yet, it was not possible to compute the case using the second-order LD-Rusanov-MUSCL formulation.

Ongoing works are to pursue comparative analysis and to develop high-order schemes for cavitating flows.

\section{Acknowledgments}

The authors gratefully thank K. Tang and A. Beccantini from the Commissariat à l'Energie Atomique for having provided the numerical solutions computed with their sevenequation model. The second author would like to particularly acknowledge the support provided by the German Jordanian University through the project SEED-SNRE 7-2014.

\section{Appendix A: Splitting functions for the AUSM+up scheme}

The splitting functions for Mach numbers and pressures are defined as polynomials, for which the numerals in the subscripts indicate the degree of the polynomials:

$$
\begin{aligned}
& M_{(1)}^{ \pm}=\frac{1}{2}(M \pm|M|), \\
& M_{(4)}^{ \pm}= \begin{cases} \pm \frac{1}{4}(M \pm 1)^{2}\left(1+\frac{1}{2}(M \mp 1)^{2}\right) & \text { if }|M|<1, \\
M_{(1)}^{ \pm} & \text {otherwise, }\end{cases} \\
& P_{(5)}^{ \pm}= \begin{cases}\frac{1}{4}(M \pm 1)^{2}(2 \mp M) \pm \frac{3}{16} M\left(M^{2}-1\right)^{2} & \text { if }|M|<1, \\
\frac{M_{1}^{ \pm}}{M} & \text { otherwise. }\end{cases}
\end{aligned}
$$




\section{Appendix B: Matrix and eigenvectors for the VF Roe ncv scheme}

The matrix $B$ is expressed as:

$$
B=\left[\begin{array}{cccc}
u & -\tau & 0 & 0 \\
0 & u & \tau & 0 \\
0 & \rho c^{2} & u & 0 \\
0 & 0 & 0 & u
\end{array}\right]
$$

The associated right eigenvectors are:

$$
\tilde{r}_{1}=\left(\begin{array}{c}
\tilde{\tau} \\
\tilde{c} \\
-\tilde{\rho} \tilde{c}^{2} \\
0
\end{array}\right), \quad \tilde{r}_{2}=\left(\begin{array}{l}
1 \\
0 \\
0 \\
0
\end{array}\right), \quad \tilde{r}_{3}=\left(\begin{array}{l}
0 \\
0 \\
0 \\
1
\end{array}\right), \quad \tilde{r}_{4}=\left(\begin{array}{c}
\tilde{\tau} \\
-\tilde{c} \\
-\tilde{\rho} \tilde{c}^{2} \\
0
\end{array}\right) .
$$

And the left eigenvectors:

$$
{ }^{t} \tilde{l}_{1}=\left(\begin{array}{c}
0 \\
\frac{1}{2 \tilde{c}_{\tilde{\tau}}} \\
-\frac{\tilde{\tau} \tilde{c}^{2}}{0}
\end{array}\right), \quad{ }^{t} \tilde{l}_{2}=\left(\begin{array}{c}
1 \\
0 \\
\frac{\tilde{\tau}}{2 \tilde{c}^{2}} \\
0
\end{array}\right), \quad{ }^{t} \tilde{l}_{3}=\left(\begin{array}{l}
0 \\
0 \\
0 \\
1
\end{array}\right), \quad{ }^{t} \tilde{l}_{4}=\left(\begin{array}{c}
0 \\
-\frac{1}{2 \tilde{c}} \\
-\frac{\tilde{\tau}}{2 \tilde{c}^{2}} \\
0
\end{array}\right) .
$$

\section{Appendix C: A barotropic model}

We consider the sinusoidal barotropic model [41] extended for compressible solver [14]. When the pressure becomes smaller than the quantity $P_{\text {vap }}+\Delta P$ and greater than $P_{\text {vap }}-$ $\Delta P$, we have the relation:

$$
P(\alpha)=P_{\text {vap }}+\left(\frac{\rho_{l}^{\text {sat }}-\rho_{v}^{\text {sat }}}{2}\right) c_{\text {baro }}^{2} \operatorname{Arcsin}(A(1-2 \alpha))
$$

where $\Delta P$ represents the pressure width of the law and, for a void ratio value of 0.5 , the pressure is equal to the saturation pressure $P_{\text {vap }}$. This law involves an adjustable parameter $c_{b a r o}$, which can be interpreted as the minimum speed of sound in the mixture. The constant $A$, close to 1 , is introduced to avoid infinite value of the speed of sound. The continuity of both the pressure and the speed of sound is ensured and determines the value of $c_{\text {baro }}$ for given values of the saturation conditions (see [14]). For the tube case, $\rho_{l}^{\text {sat }}=1150 \mathrm{~kg} / \mathrm{m}^{3}$ and $\rho_{v}^{\text {sat }}=0.31 \mathrm{~kg} / \mathrm{m}^{3}$, which lead to $c_{\text {baro }}=1.31 \mathrm{~m} / \mathrm{s}$. 


\section{Appendix D: A Kunz-type void ratio transport model}

A class of cavitation models introduces a mass transfer between phases involving a separate contribution for vaporization and condensation processes. Two tunable parameters are associated for each process. Different formulations and sets of parameters are presented in [11]. In this study, we consider a formulation derived from the model proposed by Kunz et al. [9]:

$$
\begin{aligned}
& \frac{\partial \alpha}{\partial t}+\vec{V} \cdot \operatorname{grad}(\alpha)=\frac{\dot{m}}{\rho_{l}}, \\
& \dot{m}=\dot{m}^{+}+\dot{m}^{-}=C_{\text {prod }} \rho_{v}(1-\alpha)^{2} \alpha \frac{\operatorname{Min}\left(0, P-P_{\text {vap }}\right)}{0.5 \rho_{\text {ref }} U_{\text {ref }}^{2}}+C_{\text {des }} \rho_{v}(1-\alpha) \frac{\operatorname{Max}\left(0, P-P_{\text {vap }}\right)}{0.5 \rho_{\text {ref }} U_{\text {ref }}^{2}},
\end{aligned}
$$

where $C_{\text {prod }}$ and $C_{\text {des }}$ are constants to calibrate.

With this model, the void ratio value can be higher than one. We added a limiter in the solver to clip the void ratio into its physical domain of evolution.

\section{References}

[1] M.R. Baer and J.W. Nunziato. A two-phase mixture theory for the deflagration-to-detonation transition (DDT) in reactive granular materials. Int. Journal of Multiphase Flow, 12:861-889, 1986.

[2] S.A. Tokareva and E. Toro. HLLC-type Riemann solver for the Baer-Nunziato equations of compressible two-phase flow. Journal of Computational Physics, 229:3573-3604, 2010.

[3] M. Dumbser and E.F. Toro. A simple extension of the Osher Riemann solver to nonconservative hyperbolic systems. Journal of Scientific Comp., 48:70-88, 2011.

[4] A. Ambroso, C. Chalons, and P-A. Raviart. A Godunov-type method for the seven-equation model for compressible two-phase flow. Computers \& Fluids, 54:67-91, 2012.

[5] D. Zeidan. Assessment of mixture two-phase flow equations for volcanic flows using Godunov-type methods. Applied Mathematics and Computation, 272:707-719, 2016.

[6] A.K. Kapila, R. Menikoff, J.B. Bdzil, S.F. Son, and D.S. Stewart. Two-phase modeling of deflagration-to-detonation transition in granular materials: reduced equations. Physics of fluids, 13(10):3002-3024, 2001.

[7] A. Murrone and H. Guillard. A five equation reduced model for compressible two phase flows problems. Journal of Computational Physics, 202(2):664-698, 2005.

[8] F. Daude, P. Galon, Z. Gao, and E. Blaud. Numerical experiments using a HLLC-type scheme with ALE formulation for compressible two-phase flows five-equation models with phase transition. Computer \& Fluids, 94:112-138, 2014.

[9] RF. Kunz, DA. Boger, DR. Stinebring, TS. Chyczewski, JW. Lindau, HJ. Gibeling, S. Venkateswaran, and TR. Govindan. A preconditioned Navier-Stokes method for twophase flows with application to cavitation prediction. Computers \& Fluids, 29(8):849-875, 2000.

[10] E. Goncalves and B. Charriere. Modelling for isothermal cavitation with a four-equation model. International Journal of Multiphase Flow, 59:54-72, 2014.

[11] Y. Utturkar, J. Wu, G. Wang, and W. Shyy. Recent progress in modelling of cryogenic cavitation for liquid rocket propulsion. Progress in Aerospace Sciences, 41:558-608, 2005. 
[12] E. Goncalves and R. Fortes Patella. Constraints on equation of state for cavitating flows with thermodynamic effects. Applied Math. and Computation, 217:5095-5102, 2011.

[13] S. Clerc. Numerical simulation of the homogeneous equilibrium model for two-phase flows. Journal of Computational Physics, 161(1):354-375, 2000.

[14] E. Goncalves and R. Fortes Patella. Numerical simulation of cavitating flows with homogeneous models. Computers \& Fluids, 38(9):1682-1696, 2009.

[15] J.G. Zheng, B.C. Khoo, and Z.M. Hu. Simulation of wave-flow-cavitation interaction using a compressible homogenous flow method. Commun. Comput. Phys, 14(2):328-354, 2013.

[16] R. Abgrall. How to prevent pressure oscillations in multicomponent flow calculations : a quasi conservative approach. Journal of Computational Physics, 125(1):150-160, 1996.

[17] E. Goncalves. Modeling for non isothermal cavitation using 4-equation models. International Journal of Heat and Mass Transfer, 76:247-262, 2014.

[18] A. Jameson, W. Schmidt, and E. Turkel. Numerical solution of the Euler equations by finite volume methods using Runge-Kutta time stepping schemes. In AIAA Paper 81-1259, 1981.

[19] V.V. Rusanov. Calculation of interaction of non-steady shock waves with obstacles. Journal of Computational Mathematics and Physics, 1:267-279, 1961.

[20] CH. Chang and MS. Liou. A robust and accurate approach to computing compressible multiphase flow: stratified flow model and AUSM+-up scheme. Journal of Computational Physics, 225(1):840-873, 2007.

[21] T. Gallouet, J.M. Herard, and N. Seguin. A hybrid scheme to compute contact discontinuities in Euler systems. Mathematical Modelling and Numerical Analysis, 36(6):1133-1159, 2002.

[22] EF. Toro. Riemann solvers and numerical methods for fluid dynamics. 2nd edition, New York: Springer, 1999.

[23] O. Le Metayer, J. Massoni, and R. Saurel. Elaborating equations of state of a liquid and its vapor for two-phase flow models. Int. Journal of Thermal Sciences, 43:265-276, 2004.

[24] R. Saurel, F. Petitpas, and R. Abgrall. Modelling phase transition in metastable liquids: application to cavitating and flashing flows. Journal of Fluid Mechanics, 607:313-350, 2008.

[25] G. Wallis. One-dimensional two-phase flow. New York: McGraw-Hill, 1967.

[26] E. Goncalves. Numerical study of expansion tube problems: Toward the simulation of cavitation. Computers \& Fluids, 72:1-19, 2013.

[27] D. Zeidan. Validation of hyperbolic model for two-phase flow in conservative form. Int. Journal of Computational Fluid Dynamics, 23:623-641, 2009.

[28] R. Saurel, P. Boivin, and O. Le Metayer. A general formulation for cavitating, boiling and evaporating flows. Computer \& Fluids, 128:5364, 2016.

[29] JR. Edwards and RK. Franklin. Low-diffusion flux splitting methods for real fluid flows with phase transition. AIAA Journal, 38(9):1624-1633, 2000.

[30] H. Paillere, C. Corre, and JR. Garcia Cascales. On the extension of the AUSM+ scheme to compressible two-fluid models. Computers \& Fluids, 32(6):891-916, 2003.

[31] Y.Y. Niu, Y.M. Lin, and Y.C. Lin. A simple and robust advection upwind fluw splitting to simulate transient cavitated water-vapor flows. Numerical Heat Transfer, Part A, 51:679-696, 2007.

[32] K. Kitamura, M-S. Liou, and C-H. Chang. Extension and comparative study of AUSMfamily schemes for compressible multiphase flow simulations. Commun. Comput. Phys, 16(3):632-674, 2014.

[33] M. Bilanceri, F. Beux, and M.V. Salvetti. An implicit low-diffusive HLL scheme with complete time linearization: application to cavitating barotropic flows. Computers $\mathcal{E}$ Fluids, 39:1990-2006, 2010. 
[34] E. Toro, M. Spruce, and W. Speares. Restoration of the contact surface in the HLL-Riemann solver. Shock Waves, 4:25-34, 1994.

[35] P. Batten, N. Clarke, C. Lambert, and D.M. Causon. On the choice of wave speeds for the HLLC Riemann solver. SIAM J. Sci. Comput., 18(6):1553-1570, 1997.

[36] T. Buffard, T. Gallouet, and J.M. Herard. A sequel to a rough Godunov scheme: application to real gases. Computers $\mathcal{E}$ Fluids, 29(7):813-847, 2000.

[37] T. Gallouet, J.M. Herard, and N. Seguin. Some recent finite volume schemes to compute Euler equations using real gas EOS. Int. Journal for Numerical Methods in Fluids, 39:1073$1138,2002$.

[38] R. Abgrall and R. Saurel. Discrete equations for physical and numerical compressible multiphase mixtures. Journal of Computational Physics, 186(2):361-396, 2003.

[39] F. Petitpas, E. Franquet, R. Saurel, and O. Le Metayer. A relaxation-projection method for compressible flows. Part II: artificial heat exchanges for multiphase shocks. Journal of Computational Physics, 225(2):2214-2248, 2007.

[40] A. Zein, M. Hantke, and G. Warnecke. Modeling phase transition for compressible twophase flows applied to metastable liquids. Journal of Computational Physics, 229(8):2964-2998, 2010.

[41] Y. Delannoy and JL. Kueny. Two phase flow approach in unsteady cavitation modelling. In Cavitation and Multiphase Flow Forum, ASME-FED, vol. 98, pp.153-158, 1990.

[42] G.J. Ball, B.P. Howell, T.G. Leighton, and M.J. Schofield. Shock-induced collapse of a cylindrical air cavity in water: a Free-Lagrange simulation. Shock Waves, 10:265-276, 2000.

[43] R.R. Nourgaliev, T.N. Dinh, and T.G. Theofanous. Adaptive characteristics-based matching for compressible multifluid dynamics. Journal of Computational Physics, 213:500-529, 2006.

[44] D. Ranjan, J. Oakley, and R. Bonazza. Shock-bubble interactions. Annual review of Fluid Mechanics, 43:117-140, 2011.

[45] N.A. Hawker and Y. Ventikos. Interaction of a strong shockwave with a gas bubble in a liquid mediam: a numerical study. Journal of Fluid Mechanics, 701:59-97, 2012.

[46] M. Ozlem, D.W. Schwendeman, A.K. Kapila, and W.D. Henshaw. A numerical study of shock-induced cavity collapse. Shock Waves, 22:89-117, 2012.

[47] T.G. Liu, B.C. Khoo, and K.S. Yeo. The simulation of compressible multi-medium flow, II: applications to 2D underwater shock refraction. Computers and Fluids, 30:219-314, 2001.

[48] W.F. Xie, T.G. Liu, and B.C Khoo. The simulation of cavitating flows induced by underwater shock and free surface interaction. Applied Numerical Mathematics, 57:734-745, 2007. 